\title{
LINEAR RANDOM BOUNDARY VALUE PROBLEMS CONTAINING WEAKLY CORRELATED FORCING FUNCTIONS
}

\author{
NING-MAO XIA \\ East China Institute of Chemical Technology \\ Shangha1, Peoples Republic of China
}

(Received March 20, 1984)

ABSTRACT. This paper concerns linear random boundary value problems that contain random variables in the boundary conditions and weakly correlated processes in the differential equations. When the correlation length $\varepsilon$ is small the structure of the solution is pointed out, and the formulas for the density function of the solutions are derived. The discussion is given in terms of second order equations, but extensions to higher order problems are readily apparent.

KEY WORDS AND PHRASES; Random Boundary Value Problems, Differential Equations, Stochastic Differential Equations, Density Functions, Asymptotic Approximations.

1980 AMS SUBJECT CLASSIFICATION CODES. 34F05, 34K10.

1. INTRODUCTION.

For many years it has been of interest to find conditions under which the distribution of the solution of a random differential equation tends to a normal distribution.

In 1930, while studying Brownian motion, Uhlenbeck and Ornstein [1] established that the solution $x(t)$ of certain initial value problems has approximately a normal distribution.

In 1966 Boyce [2] obtained a similar result for a class of linear self-adjoint boundary value problems

$$
\mathrm{L}[\mathrm{x}]=\mathrm{f}(\mathrm{t}), \quad 0<\mathrm{t}<1
$$

with boundary conditions

$$
\mathrm{U}_{\mathbf{i}}[\mathrm{x}]=0 \text {, }
$$

at the end points. The operator $L$ has the form

$$
L[x]=\sum_{j=0}^{m}(-1)^{j}\left[\gamma_{j}(t) x^{(j)}\right](j) \text {. }
$$

Randomness entered the problem (1.1), (1.2) only through the forcing function $f$, which we assumed to be weakly correlated with correlation length $\varepsilon \ll 1$. The solution $y(t)$ has a property that the distribution function of $y(t) / \sqrt{\varepsilon}$ approaches normal as $\varepsilon+0$. 
When $f, \gamma_{0}, \ldots, \gamma_{m-1}, \gamma_{m}$ are small independent weakly correlated processes, Purkert and vom Scheidt [3,4]; Boyce and Xia [5] found a similar and better results by combining the methods of [2] with perturbation and Chebyshev-Hermite polynomial expansion.

Here we extend the results of [2] in another way, our problem need not be selfadjoint and the random parts of the forcing terms need not be small.

When $\gamma_{m}(x) \neq 0$ we can always rewrite equation (1.1) into a standard linear system, so instead of (1.1) in this paper we consider a general linear system with random boundary conditions and with weakly correlated processes in the forcing terms.

In Section 2 we will define the problem and derive the functional form of the solution. Section 3 contains some preliminary results that are required later. The main results of this paper are in Section 4 , that is when $\varepsilon+0$ we can find the asymptotic approximation for the density function of the solution which has a nice structure, namely it consists of three functions, one is deterministic; one can be obtained in terms of the random boundary conditions; and the other, which is the contribution of the random forcing terms, has the normal distribution. The usefulness of this expression is illustrated by the examples in Section 5 .

2. STATEMENT OF THE PROBLEM AND THE FORM OF THE SOLUTION.

Although the same methods can be applied to higher order problems, in this section we consider the linear random boundary value problem of the second order differential equation.

$$
\begin{aligned}
& \frac{d \underset{\sim}{X}(t)}{d t}=\underset{\sim}{A}(t) \underset{\sim}{X}(t)+\underset{\sim}{B}(t) \xi(t, \omega)+\underset{\sim}{C}(t) \\
& \mathbb{\sim}_{1} \underset{\sim}{x}(0)+D_{2} \underset{\sim}{X}(1)=\underset{\sim}{\alpha}(\omega),
\end{aligned}
$$

where $\underset{\sim}{\mathrm{A}}(\mathrm{t}), \underset{\sim}{\mathrm{B}}(\mathrm{t}), \underset{\sim}{\mathrm{D}} 1, \underset{\sim}{\mathrm{D}} 2$ are deterministic $2 \times 2$ matrices

$$
\begin{aligned}
\underset{\sim}{A}(t)=\left(\begin{array}{ll}
a_{11}(t) & a_{12}(t) \\
a_{21}(t) & a_{22}(t)
\end{array}\right), & \underset{\sim}{B}(t)=\left(\begin{array}{ll}
b_{11}(t) & b_{12}(t) \\
b_{21}(t) & b_{22}(t)
\end{array}\right) \\
D_{1}=\left(\begin{array}{ll}
d_{1}^{(1)} & d_{12}^{(1)} \\
d_{21}^{(1)} & d_{22}^{(1)}
\end{array}\right), & D_{2}=\left(\begin{array}{ll}
d_{11}^{(2)} & d_{12}^{(2)} \\
d_{21}^{(2)} & d_{22}^{(2)}
\end{array}\right)
\end{aligned}
$$

$\underset{\sim}{C}(t), \underset{\sim}{x}(t)$ are $2 \times 1$ vectors

$$
\underset{\sim}{C}(t)=\left(C_{1}(t), C_{2}(t)\right)^{T} \quad \underset{\sim}{ }(t)=(x(t), y(t))^{T},
$$

and $\xi(t, \omega), \underset{\sim}{\alpha}(\omega)$ are random $2 \times 1$ vectors defined on an underlying probability space $(\Omega, F, P)$

$$
\underset{2}{\xi}(t, \omega)=\left(\xi_{1}(t, \omega), \xi_{2}(t, \omega)\right)^{\mathrm{T}}, \underset{\sim}{\alpha}(\omega)=(\alpha(\omega), \beta(\omega))^{\mathrm{T}} .
$$

We are interested in the case in which $\xi_{1}(t, \omega)$ and $\xi_{1}(t, \omega)$ and $\xi_{2}(t, \omega)$ are weakly correlated with the same correlation length $\varepsilon$. The term "weakly correlated" has been defined by Purkert and vom Scheidt $[3,6,4]$ in the following way. Let $S=$ $\left(t_{1}, t_{2}, \ldots, t_{n}\right)$ be an $n$-tuple of real numbers and let $\varepsilon>0$ be a positive constant. 
Let $s_{1}=\left(t_{i 1}, t_{i 2}, \ldots, t_{i k}\right)$ be a subset of $s$, and suppose that $t_{i 1} \leq t_{i 2} \leq \cdots \leq t_{i k}$; this ordering can always be attained by relabeling the elements of $S_{1}$ if necessary. Then $S_{1}$ is said to be $\varepsilon$-neighboring if

$$
\left|t_{i 1}-t_{i 2}\right| \leq \varepsilon,\left|t_{i 2}-t_{i 3}\right| \leq \varepsilon, \ldots\left|t_{i k}-1-t_{i k}\right| \leq \varepsilon
$$

A single element subset is always $\varepsilon$-neighboring. The subset $S_{1}$ is maximally $\varepsilon$-neighboring, with respect to $S$, if $S_{1}$ is $\varepsilon$-neighboring but is not contained in any larger $\varepsilon$-neighboring subset of $S$. It can be shown [6] that $S$ can be separated into disjoint maximally $\varepsilon$-neighboring subsets in a unique way. Then a stochastic process $h(t, w)$ is said to be weakly correlated with correlation length $\varepsilon$ if, for each $\mathrm{n}$,

$$
\left\langle h\left(t_{1}, w\right) \ldots h\left(t_{n}, w\right)\right\rangle=\left\langle h\left(t_{11}, w\right) \ldots h\left(t_{1 p l}, w\right)\right\rangle \ldots\left\langle h\left(t_{k l}, w\right) \ldots h\left(t_{k p_{k}}, w\right)\right\rangle
$$

where $\langle$.$\rangle denotes the mathematical expectation and the n$-tuple $S$ has been separated into the maximally $\varepsilon$-neighboring subsets $\left(t_{1}, \ldots, t_{1 p_{1}}\right), \ldots,\left(t_{k l}, \ldots, t_{k p_{k}}\right)$ with $\sum_{i=1}^{k} p_{i}=n$.

In this paper we assume that $\xi_{1}(t, \omega)$ and $\xi_{2}(t, \omega)$ have the property $(2.5)$, and without loss of generality we also assume that

$$
\left\langle\xi_{1}(t, \omega)\right\rangle=0, \quad\left\langle\xi_{2}(t, w)\right\rangle=0
$$

Otherwise we can redefine $\underset{\sim}{\mathrm{C}}(t)$ so as to include $\langle\xi(t, \omega)\rangle$.

For the case $\mathrm{n}=2$ the condition $(2.5)$ reduces to

$$
\left\langle\xi_{i}\left(t_{1}, \omega\right) \xi_{i}\left(t_{2}, \omega\right)\right\rangle=\left\{\begin{array}{l}
0,\left|t_{1}-t_{2}\right|>\varepsilon \\
R_{\xi_{i}}\left(t_{1}, t_{2}\right), \quad\left|t_{1}-t_{2}\right| \leq \varepsilon
\end{array} \quad i=1.2 .\right.
$$

Now we consider the form of the solution of problem $(2 \cdot 1)$. Let $\underset{\sim}{p}(t)$ be the fundamental matrix of the homogeneous system corresponding to (2.1). Then $\underset{\sim}{\Phi}(t)$ satisfies

$$
\frac{d \Phi}{d t}=\underset{\sim}{A}(t) \stackrel{D}{\sim}(t), \quad \stackrel{\mathbb{D}}{\sim}(0)=\underset{\sim}{I}
$$

where $\underset{\sim}{I}$ is the $2 \times 2$ identity matrix, $\underset{\sim}{D}(t)=\left(\phi_{i j}(t)\right)$ is a uniquely determined and nonsingular $2 \times 2$ matrix.

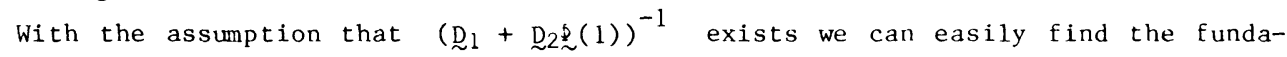
mental matrix of the homogeneous system corresponding to $(2.1$ a) and the boundary condition $(2.1$ b). Let this fundamental matrix be $\underset{\sim}{\Psi}(t)$, then $\underset{\sim}{\Psi}(t)$ satisfies

$$
\frac{\mathrm{d} \underset{\sim}{\Psi}(\mathrm{t})}{\mathrm{d} \mathrm{t}}=\underset{\sim}{\mathrm{A}}(\mathrm{t}) \underset{\sim}{\Psi}(\mathrm{t}), \quad \mathrm{D}_{1} \underset{\sim}{\Psi}(0)+\mathbb{D}_{2} \underset{\sim}{\Psi}(1)=\underset{\sim}{\mathrm{I}}
$$

and is given by

$$
\underset{\sim}{\Psi}(t)=\underset{\sim}{\Psi}(t)\left[D_{1}+D_{2} \Phi(1)\right]^{-1} \text {. }
$$

In order to find the solution of $(2 \cdot 1)$, we set 


$$
\underset{\sim}{x}(t)=\psi(t) \underset{\sim}{K}(t)
$$

where $\underset{N}{K}(t)=\left(k_{1}(t), K_{2}(t)\right)^{T}$ is a $2 \times 1$ vector to be determined. If we substitute $(2.11)$ into $(2.1$ a) and use (2.9), we have

$$
\underset{\sim}{\Psi}(t) \frac{d \underset{N}{ }(t)}{d t}=\underset{\sim}{B}(t) \xi(t, w)+\underset{\sim}{C}(t) .
$$

In the case $\left[D_{1}+D_{2} \sim_{(1)}(1)\right]^{-1}$ exists we find that

$$
\begin{aligned}
\frac{d \underset{\sim}{d}(t)}{d t} & ={\underset{\sim}{\Psi}}^{-1}(t)[\underset{\sim}{B}(t) \xi(t, \omega)+\underset{\sim}{C}(t)] \\
& =\left[\underset{\sim}{D_{1}}+\underset{\sim}{D_{2}} \underset{\sim}{\mathscr{Z}}(1)\right] \underset{\sim}{\mathbb{P}}(t)^{-1}[\underset{\sim}{B}(t) \xi(t, \omega)+\underset{\sim}{C}(t)],
\end{aligned}
$$

and then

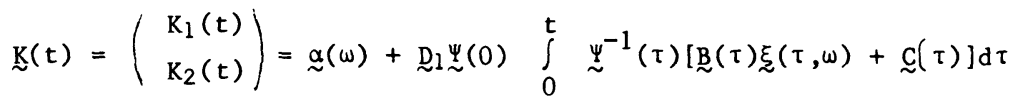

$$
\begin{aligned}
& -\mathbb{D}_{2} \underset{\sim}{\Psi}(1) \int_{t}^{1}{\underset{\sim}{\Psi}}^{-1}(\tau)[\underset{\sim}{B}(\tau) \xi(\tau, \omega)+\underset{\sim}{C}(\tau)] d \tau \\
& \underset{\sim}{X}(t)=\underset{\sim}{\Psi}(t)\left[\underset{\sim}{\alpha}(\omega)+\mathbb{D}_{1} \underset{\sim}{\Psi}(0) \int_{0}^{t}{\underset{\sim}{\Psi}}^{-1}(\tau)[\underset{\sim}{B}(\tau) \xi(\tau, \omega)+\underset{\sim}{C}(\tau)] d \tau\right. \\
& \left.-D_{\sim} \underset{\sim}{\Psi}(1) \int_{t}^{1} \underset{\sim}{\Psi}-1(\tau)[\underset{\sim}{B}(\tau) \xi(\tau, \omega)+\underset{\sim}{C}(\tau)] d \tau\right]
\end{aligned}
$$

where the boundary condition $(2.1 \mathrm{~b})$ has been used to obtain the last two results.

3. SOME PRELIMINARY RESULTS.

In order to obtain the density function of the solution $\underset{d}{x}(t)$, we first rewrite it into the following form

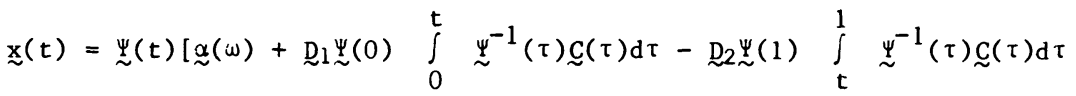

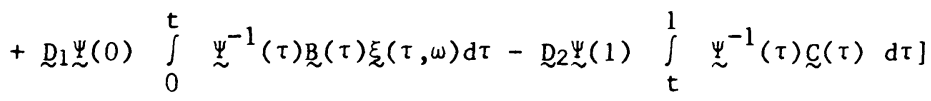

$$
\begin{aligned}
& =\underset{\sim}{\Psi}(t) \underset{\sim}{\alpha}(\omega)+\underset{\sim}{\delta}(t)+\chi(t, \omega),
\end{aligned}
$$

where

$$
\begin{aligned}
\underset{\sim}{\delta}(t) & =\left(\delta_{1}(t), \delta_{2}(t)\right)^{T} \\
& =\underset{\sim}{\Psi}(t) \underset{\sim}{D_{\sim}} \underset{\sim}{\Psi}(0) \int_{0}^{t}{\underset{\sim}{\Psi}}^{-1}(\tau) \underset{\sim}{\mathcal{C}}(\tau) \mathrm{d} \tau-\underset{\sim}{\Psi}(t) \underset{2}{D_{2}} \underset{\sim}{\Psi}(1) \int_{t}^{1}{\underset{\sim}{\Psi}}^{-1}(\tau) \underset{\sim}{\mathcal{C}}(\tau) \mathrm{d} \tau
\end{aligned}
$$

is a deterministic $2 \times 1$ vector, and

$$
\begin{aligned}
\mathfrak{X}(t, \omega)= & \left(\gamma_{1}(t, \omega), \gamma_{2}(t, \omega)\right)^{T} \\
= & \underset{\sim}{\Psi}(t) \underset{\sim}{D_{1}} \underset{\sim}{\Psi}(0) \int_{0}^{t} \underset{\sim}{\Psi}(\tau) \underset{\sim}{B}(\tau) \underset{\sim}{\xi}(\tau, \omega) \mathrm{d} t \\
& \quad-\underset{\sim}{\Psi}(t) \underset{\sim}{D_{2}} \underset{\sim}{\Psi}(1) \int_{t}^{1} \underset{\sim}{\Psi}-1(\tau) \underset{\sim}{B}(\tau) \xi(\tau, \omega) \mathrm{d} \tau
\end{aligned}
$$

is a random $2 \times 1$ vector. 
If we set

then

$$
\begin{aligned}
& \underset{\sim}{\Psi}(t) \underset{\sim}{D_{1}} \underset{\sim}{\Psi}(0) \underset{\sim}{\Psi}(\tau) \underset{\sim}{\mathrm{B}}(\tau)=\left(\begin{array}{ll}
\mathrm{n}_{1}(\tau) & \mathrm{r}_{1}(\tau) \\
\mathrm{n}_{3}(\tau) & \mathrm{r}_{3}(\tau)
\end{array}\right) ; \\
& -\underset{\sim}{\Psi}(\mathrm{t}) \underset{\sim}{\mathrm{D}_{2} \underset{\sim}{\Psi}(1) \underset{\sim}{\Psi}-1}(\tau) \underset{\sim}{\mathrm{B}}(\tau)=\left(\begin{array}{ll}
\mathrm{n}_{2}(\tau) & \mathrm{r}_{2}(\tau) \\
\mathrm{n}_{4}(\tau) & \mathrm{r}_{4}(\tau)
\end{array}\right)
\end{aligned}
$$

where

$$
\chi(t, \omega)=\left(\begin{array}{l}
\gamma_{1}(t, \omega) \\
\gamma_{2}(t, \omega)
\end{array}\right)=\left(\begin{array}{l}
n_{1}(t, \omega)+n_{2}(t, \omega) \\
n_{3}(t, \omega)+n_{4}(t, \omega)
\end{array}\right),
$$

$$
\begin{aligned}
& n_{1}(t, \omega)=\int_{0}^{t}\left[n_{1}(\tau) \xi_{1}(\tau, \omega)+r_{1}(\tau) \xi_{2}(\tau, \omega)\right) d \tau, \\
& n_{2}(t, \omega)=\int_{t}^{1}\left[n_{2}(\tau) \xi_{1}(\tau, \omega)+r_{2}(\tau) \xi_{2}(\tau, \omega)\right] d \tau, \\
& n_{3}(t, \omega)=\int_{0}^{t}\left[n_{3}(\tau) \xi_{1}(\tau, \omega)+r_{3}(\tau) \xi_{2}(\tau, \omega)\right] d \tau, \\
& n_{4}(t, \omega)=\int_{t}^{1}\left[n_{4}(\tau) \xi_{1}(\tau, \omega)+r_{4}(\tau) \xi_{2}(\tau, \omega)\right] d \tau,
\end{aligned}
$$

From now on we always assume that $\xi_{1}(t, \omega)$ and $\xi_{2}(t, \omega)$ are independent weakly correlated processes with the same correlation length $\varepsilon$, then we can show that as $\varepsilon \rightarrow 0, n_{1}(t, \omega)$ and $n_{2}(t, \omega) ; n_{3}(t, \omega)$ and $n_{4}(t, w)$ will be independent and they will have the normal distributions.

In order to prove this and then to obtain the density function of $x(t)$ and $y(t)$, the following properties are required.

[Property 1] If we have

$$
\begin{aligned}
& A_{1}(t)=2 \int_{0}^{t}\left[n_{1}^{2}(\tau) R_{\xi_{1}}(\tau, \tau)+r_{1}^{2}(\tau) R_{\xi_{2}}(\tau, \tau)\right] d \tau \neq 0 \\
& B_{l}(t)=2 \int_{t}^{1}\left[n_{2}^{2}(\tau) R_{\xi_{1}}(\tau, \tau)+r_{2}^{2}(\tau) R_{\xi_{2}}(\tau, \tau)\right] d \tau \neq 0
\end{aligned}
$$

then for $n=1,2, \ldots$

$$
\begin{aligned}
& <\left(\frac{n_{1}}{\sqrt{A_{1} \varepsilon}}\right)^{2 n}>=\frac{(2 n) !}{2^{n} n !}+0(\varepsilon) \\
& <\left(\frac{n_{1}}{\sqrt{A_{1} \varepsilon}}\right)^{2 n+1}>=\frac{(2 n+1) !}{3 ! 2^{n-1}(n-1) !} \cdot \frac{A_{3}}{A_{1}^{3 / 2}} \varepsilon^{1 / 2}+0\left(\varepsilon^{3 / 2}\right) \\
& <\left(\frac{n_{2}}{\sqrt{B_{1} \varepsilon}}\right)^{2 n}>=\frac{(2 n) !}{2^{n} n !}+0(\varepsilon) \\
& <\left(\frac{n_{2}}{\sqrt{B_{1} \varepsilon}}\right)^{2 n+1}>=\frac{(2 n+1) !}{3 ! 2^{n-1}(n-1) !} \cdot \frac{B_{3}}{B_{1}^{3 / 2}} \varepsilon^{1 / 2}+0\left(\varepsilon^{3 / 2}\right)
\end{aligned}
$$

where $A_{3}(t), B_{3}(t)$ are the corresponding main parts of $\left\langle n_{1}^{3}\right\rangle$ and $\left\langle n_{2}^{3}\right\rangle$ respectively, and $O\left(\varepsilon^{\mathrm{m}}\right)$ denotes the term with the order $d \geq \mathrm{m}$ (see $\left.[5,2]\right)$. 
[Property 2] If $\mathrm{K}_{1}, \mathrm{~K}_{2}=1,2, \ldots$ and $\left(\tau_{1}, \ldots, \tau_{\mathrm{K}_{1}}+\mathrm{K}_{2}\right)$ is $\varepsilon$-neighboring then

$$
\underbrace{\int_{0}^{\mathrm{t}} \ldots \int_{0}^{t}}_{\mathrm{K}_{1}} \underbrace{\int_{\mathrm{t}}^{1} \ldots \int_{1}^{1}}_{\mathrm{K}_{2}}[\ldots] d \tau_{1} \ldots d \tau_{K_{1}}+\mathrm{K}_{2}=0\left(\varepsilon^{\mathrm{K}_{1}+\mathrm{K}_{2}}\right) \text {. }
$$

where the integrand [...] is any function having continuous derivatives, and $t$ is any fixed number between 0 and 1 .

Proof: Consider the case $K_{1}=2, K_{2}=1$ for simplicity. Then the region of integration is

$$
G_{3}=\left\{\tau_{1}, \tau_{2}, \tau_{3} \mid \begin{array}{l}
\tau_{1} \varepsilon[0, t], \tau_{2} \varepsilon[0, t], \tau_{3} \varepsilon[t, 1] \\
\left.\left(\tau_{1}, \tau_{2}, \tau_{3}\right) \text { is } \varepsilon \text {-neighboring }\right\} .
\end{array}\right.
$$

From the definition of $\varepsilon$-neighboring we know that if $\tau_{2} \geq \tau_{1}$, then $0 \leq \tau_{3}-\tau_{2} \leq \varepsilon, 0 \leq \tau_{2}-\tau_{1} \leq \varepsilon$; and if $\tau_{1} \geq \tau_{2}$ then $0 \leq \tau_{3}-\tau_{1} \leq \varepsilon$, o $\leq \tau_{1}-\tau_{2} \leq \varepsilon$. The region $G_{3}$ can be divided into two parts

$$
G_{3}=G_{3}^{(1)} \bigcup_{G_{3}^{(2)}}^{(2)}
$$

where

$$
\begin{gathered}
G_{3}^{(1)}=\left\{\tau_{1}, \tau_{2}, \tau_{3} \mid \begin{array}{l}
\tau_{1} \varepsilon[0, t], \tau_{2} \varepsilon[0, t], \tau_{3} \varepsilon[t, 1] \\
0 \leq \tau_{3}-\tau_{2} \leq \varepsilon, 0 \leq \tau_{2}-\tau_{1} \leq \varepsilon
\end{array}\right\}
\end{gathered}
$$

and

$$
G_{3}^{(2)}=\left\{\tau_{1}, \tau_{2}, \tau_{3} \mid \begin{array}{l}
\tau_{1} \varepsilon[0, t], \tau_{2} \varepsilon[0, t], \tau_{3} \varepsilon[t, 1] \\
0 \leq \tau_{3}-\tau_{1} \leq \varepsilon, 0 \leq \tau_{1}-\tau_{2} \leq \varepsilon
\end{array}\right\} .
$$

Now we consider the distance between the point $P=\left(\tau_{1}, \tau_{2}, \tau_{3}\right)$ in $G_{3}$ and the point $T=(t, t, t)$. Because of (3.8) we may assume $P \varepsilon G_{3}^{(1)}$ and then from (3.9 a) we find that

$$
t \leq \tau_{3} \leq \tau_{2}+\varepsilon, \quad t-\varepsilon \leq \tau_{3}-\varepsilon \leq \tau_{2} \leq t, \quad t-2 \varepsilon \leq \tau_{2}-\varepsilon \leq \tau_{1} \leq t,
$$

or

$$
t \leq \tau_{3} \leq t+\varepsilon, \quad t-\varepsilon \leq \tau_{2} \leq t, \quad t-2 \varepsilon \leq \tau_{1} \leq t .
$$

An estimate for the distance $\rho(\mathrm{P}, \mathrm{T})$ is

$$
\begin{aligned}
\rho(P, T) & =\sqrt{\left(\tau_{1}-t\right)^{2}+\left(\tau_{2}-t\right)^{2}+\left(\tau_{3}-t\right)^{2}} \leq \sqrt{(2 \varepsilon)^{2}+\varepsilon^{2}+\varepsilon^{2}} \\
& =\sqrt{6 \varepsilon}=O(\varepsilon) .
\end{aligned}
$$

From this estimate we have

$$
\begin{aligned}
\mid \iiint_{G_{3}} & {[\ldots] d \tau_{1} d \tau_{2} d \tau_{3} \mid } \\
= & |[\ldots]|_{P=T} \cdot \iiint_{G_{3}} d \tau_{1} d \tau_{2} d \tau_{3}+0(\varepsilon) \iiint_{G_{3}} d \tau_{1} d \tau_{2} d \tau_{3}
\end{aligned}
$$




$$
\begin{aligned}
& \leq|[\ldots]|_{P=T} \cdot \iiint_{0_{3}} d \tau_{1} d \tau_{2} d \tau_{3}+O(\varepsilon) \iiint_{0_{3}} d \tau_{1} d \tau_{2} d \tau_{3} \\
& =O\left(\varepsilon^{3}\right)
\end{aligned}
$$

where $0_{3}$ is a ball, with center at $T=(t, t, t)$ and with $\operatorname{radius} \max (P, T) \leq \sqrt{6 \varepsilon}$. Thus we have proved (3.7); for other $K_{1}, K_{2}$ the situation is similar.

[Property 3] If $K_{1}, K_{2}=1,2, \ldots$, and $\left(\tau_{1}, \ldots, \tau_{K_{1}}+K_{2}\right)$ is $\varepsilon$-neighboring then

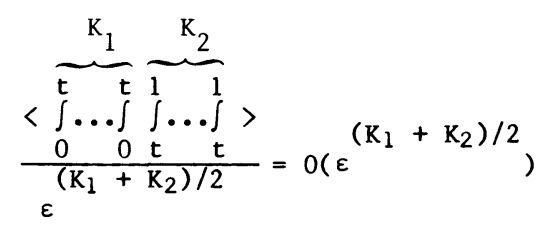

where we assume that the integrand and $t$ have the same properties as in (3.7).

[Property 4] If we assume that the coefficients of equation $\left(\begin{array}{ll}2.1 & a\end{array}\right)$ have the properties that $\underset{\sim}{A}(t) \varepsilon C^{1}[0,1], \underset{\sim}{B}(t) \varepsilon C^{1}[0,1], \underset{\sim}{C}(t) \varepsilon C^{1}[0,1]$, and all the mathematical expectations of different orders for $\xi_{1}(t, \omega), \xi_{2}(t, \omega)$ have continuous derivatives, then we have the conclusion that for $\mathrm{K}_{1}, \mathrm{~K}_{2}=0,1,2, \ldots$

$$
\left\langle\left(\frac{n_{1}}{\sqrt{A_{1} \varepsilon}}\right)^{K_{1}}\left(\frac{n_{1}}{\sqrt{B_{1} \varepsilon}}\right)^{K_{2}}\right\rangle-\left\langle\left(\frac{n_{1}}{\sqrt{A_{1} \varepsilon}}\right)^{K_{1}}\right\rangle \cdot\left\langle\left(\frac{n_{1}}{\sqrt{B_{1} \varepsilon}}\right)^{K_{2}}\right\rangle=0(\varepsilon) .
$$

Proof: For $K_{1}=0$ or $K_{2}=0(3.11)$ is obvious. Now we consider $\mathrm{K}_{1}, \mathrm{~K}_{2}=1,2, \ldots$. In order to find the contribution of $\left\langle\left(\frac{n_{1}}{\sqrt{A_{1} \varepsilon}}\right)^{K_{1}}\left(\frac{n_{2}}{\sqrt{B_{1} \varepsilon}}\right)^{K_{2}}>\right.$ we use (2.5) and consider all different maximally $\varepsilon$-neighboring subsets $\left(\tau_{11}, \ldots \tau_{1 P_{1}}\right), \ldots$, $\left(\tau_{i 1}, \ldots, \tau_{i P_{i}}\right), \ldots,\left(\tau_{K 1}, \ldots, \tau_{K P_{K}}\right)$ with $\sum_{i} \sum_{1}^{K} P_{i}=K_{1}+K_{2} \cdot$ These maximally $\varepsilon-$ neighboring subsets are of two distinct kinds. The first kind consists of all maximally $\varepsilon$-neighboring subsets for which at least one of its subsets has the property that

$\left(\tau_{i 1}, \ldots, \tau_{i p_{i}}\right)=\left(\tau_{i 1}, \ldots, \tau_{i q_{i}}, \tau_{i q_{i}+1}, \ldots, \tau_{i p_{i}}\right), \tau_{i l}, \ldots, \tau_{i q_{i}} \varepsilon[0, t]$, and $\tau_{i q_{i}+1}, \ldots, \tau_{i p_{i}} \varepsilon[t, 1]$. The second kind consists of all maximally $\varepsilon$-neighboring subsets for which every subset has the property that $\tau_{i 1}, \ldots, \tau_{i p_{i}} \varepsilon[0, t]$ or $\tau_{i 1}, \ldots, \tau_{i p_{i}} \varepsilon[t, 1]$

Because of (3.6) and (3.10), we know that all integrals on the maximally $\varepsilon$-neighboring subsets of the first kind have the order $\varepsilon$; and if we notice that all the integrals on the maximally $\varepsilon$-neighboring subsets of the second kind are just the contribution of $\left\langle\left(\frac{n_{1}}{\sqrt{A_{1} \varepsilon}}\right)^{K_{1}}\right\rangle\left\langle\left(\frac{n_{1}}{\sqrt{B_{1} \varepsilon}}\right)^{K_{2}}\right\rangle$, then we have the conclusion (3.11). For example, when $\mathrm{K}_{1}=2, \mathrm{~K}_{2}=2$, we consider 


$$
\left\langle\frac{n_{1}^{2}}{A_{1} \varepsilon} \frac{n_{2}^{2}}{B_{1} \varepsilon}\right\rangle=\frac{1}{A_{1} B_{1} \varepsilon^{2}} \int_{0}^{t} \int_{0}^{t} \int_{t}^{1} \int_{t}^{1}\langle[\ldots]\rangle d \tau_{1} d \tau_{2} d \tau_{3} d \tau_{4}
$$

where

$$
\begin{aligned}
\langle[\ldots]\rangle=\langle & {\left[n_{1}\left(\tau_{1}\right) \xi_{1}\left(\tau_{1}\right)+r_{1}\left(\tau_{1}\right) \xi_{2}\left(\tau_{1}\right)\right]\left[n_{1}\left(\tau_{2}\right) \xi_{1}\left(\tau_{2}\right)+r_{1}\left(\tau_{2}\right) \xi_{2}\left(\tau_{2}\right)\right] } \\
& {\left.\left[n_{2}\left(\tau_{3}\right) \xi_{1}\left(\tau_{3}\right)+r_{2}\left(\tau_{3}\right) \xi_{2}\left(\tau_{3}\right)\right]\left[n_{2}\left(\tau_{4}\right) \xi_{1}\left(\tau_{4}\right)+r_{2}\left(\tau_{4}\right) \xi_{2}\left(\tau_{4}\right)\right]\right\rangle . }
\end{aligned}
$$

For simplicity we only pick the first term in the integrand, that is

$$
\begin{aligned}
& \frac{1}{A_{1} B_{1} \varepsilon^{2}} \int_{0}^{t} \int_{0}^{t} \int_{t}^{1} \int_{t}^{1} n_{1}\left(\tau_{1}\right) n_{1}\left(\tau_{2}\right) n_{2}\left(\tau_{3}\right) n_{2}\left(\tau_{4}\right)\left\langle\xi_{1}\left(\tau_{1}\right) \xi_{1}\left(\tau_{2}\right) \xi_{1}\left(\tau_{3}\right) \xi_{1}\left(\tau_{4}\right)\right\rangle d \tau_{1} d \tau_{2} d \tau_{3} d \tau_{4} \\
& =\frac{1}{A_{1} B_{1} \varepsilon^{2}} \int_{0}^{t} \int_{0}^{t} \int_{t}^{1} \int_{t}^{1} n_{1}\left(\tau_{1}\right) n_{1}\left(\tau_{2}\right) n_{2}\left(\tau_{3}\right) n_{2}\left(\tau_{4}\right)\left\langle\xi_{1}\left(\tau_{1}\right) \xi_{1}\left(\tau_{2}\right) \xi_{1}\left(\tau_{3}\right) \xi_{1}\left(\tau_{4}\right)\right\rangle d \tau_{1} d \tau_{2} d \tau_{3} d \tau_{4} \\
& +\frac{1}{A_{1} B_{1} \varepsilon} \int_{0}^{2} \int_{R_{12}}^{t} n_{1}\left(\tau_{1}\right) n_{1}\left(\tau_{2}\right)\left\langle\xi_{1}\left(\tau_{1}\right) \xi_{1}\left(\tau_{2}\right)\right\rangle d \tau_{1} d \tau_{2} \cdot \int_{\substack{t \\
R_{34}}}^{t} n_{2}\left(\tau_{3}\right) n_{2}\left(\tau_{4}\right)\left\langle\xi_{1}\left(\tau_{3}\right) \xi_{1}\left(\tau_{4}\right)>d \tau_{3} d \tau_{4}\right. \\
& -\frac{1}{A_{1} B_{1} \varepsilon} \int_{0}^{t} \int_{0}^{t} \int_{t}^{1} \int_{t}^{1} n_{1}\left(\tau_{1}\right) n_{1}\left(\tau_{2}\right) n_{2}\left(\tau_{3}\right) n_{2}\left(\tau_{4}\right)\left\langle\xi_{1}\left(\tau_{1}\right) \xi_{1}\left(\tau_{2}\right)\right\rangle\left\langle\xi_{1}\left(\tau_{3}\right) \xi_{1}\left(\tau_{4}\right)\right\rangle d \tau_{1} d \tau_{2} d \tau_{3} d \tau_{4} \\
& \left(R_{12} \times R_{34}\right) \cap R_{1234} \\
& +\frac{1}{A_{1} B_{1} \varepsilon} 2 \int_{0}^{t} \int_{R_{13}}^{1} n_{1}\left(\tau_{1}\right) n\left(\tau_{3}\right)\left\langle\xi_{1}\left(\tau_{1}\right) \xi_{1}\left(\tau_{3}\right)\right\rangle d \tau_{1} d \tau_{3} \int_{0}^{t} \int_{t}^{1} n_{1}\left(\tau_{2}\right) n_{2}\left(\tau_{4}\right)\left\langle\xi_{1}\left(\tau_{2}\right) \xi_{1} \quad\left(\tau_{4}\right) d \tau_{2} d \tau_{4}\right.
\end{aligned}
$$

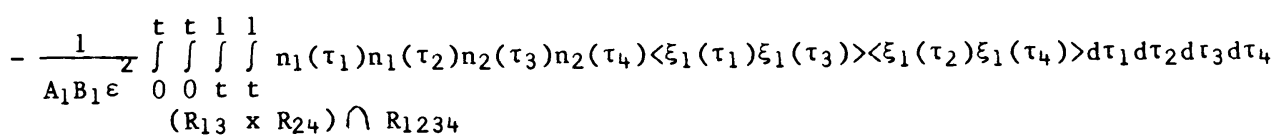

$$
\begin{aligned}
& +\frac{1}{A_{1} B_{1} \varepsilon} \int_{0}^{t} \int_{t}^{1} n_{1}\left(\tau_{1}\right) n_{2}\left(\tau_{4}\right)\left\langle\xi_{1}\left(\tau_{1}\right) \xi_{1}\left(\tau_{4}\right)\right\rangle d \tau_{1} d \tau_{4} \int_{0}^{t} \int_{t}^{1} n_{1}\left(\tau_{2}\right) n_{2}\left(\tau_{3}\right)\left\langle\xi_{1}\left(\tau_{2}\right) \xi_{1}\left(\tau_{3}\right)\right\rangle d \tau_{2} d \tau_{3} \\
& \begin{array}{l}
\left.-\frac{1}{A_{1} B_{1} \varepsilon} \int_{0}^{t} \int_{0}^{t} \int_{t}^{1} \int_{t}^{1} n_{1}\left(\tau_{1}\right) n_{1}\left(\tau_{2}\right) n_{2}\left(\tau_{3}\right) n_{2}\left(\tau_{4}\right)\left\langle\xi_{1}\left(\tau_{1}\right) \xi_{1}\left(\tau_{4}\right)\right)\right\rangle\left\langle\xi_{1}\left(\tau_{2}\right) \xi_{1} \quad\left(\tau_{3}\right)\right\rangle d \tau_{1} d \tau_{2} d \tau_{3} d \tau_{4} \\
R_{1234}
\end{array} \\
& =\frac{1}{A_{1} B_{2} \varepsilon} \int_{0}^{t} \int_{0}^{t} \int_{t}^{1} \int_{t}^{1} n_{1}\left(\tau_{1}\right) n_{1}\left(\tau_{2}\right) n_{2}\left(\tau_{3}\right) n_{2}\left(\tau_{4}\right)\left[\left\langle\xi_{1}\left(\tau_{1}\right) \xi_{1}\left(\tau_{2}\right) \xi_{1}\left(\tau_{3}\right) \xi_{1}\left(\tau_{4}\right)\right\rangle-\right. \\
& \left\langle\xi_{1}\left(\tau_{1}\right) \xi_{1}\left(\tau_{2}\right)\right\rangle\left\langle\xi_{1}\left(\tau_{3}\right) \xi_{1}\left(\tau_{4}\right)\right\rangle-\left\langle\xi_{1}\left(\tau_{1}\right) \xi_{1}\left(\tau_{3}\right)\right\rangle\left\langle\xi_{1}\left(\tau_{2}\right) \xi_{1}\left(\tau_{4}\right)\right\rangle- \\
& \left.\left\langle\xi_{1}\left(\tau_{1}\right) \xi_{1}\left(\tau_{4}\right)\right\rangle\left\langle\xi_{1}\left(\tau_{2}\right) \xi_{1}\left(\tau_{3}\right)\right\rangle\right] d \tau_{1} d \tau_{2} d \tau_{3} d \tau_{4} \\
& +\frac{1}{A_{1} B_{1} \varepsilon} \int_{0}^{t} \int_{0}^{t} n_{1}\left(\tau_{1}\right) n_{1}\left(\tau_{2}\right)\left\langle\xi_{1}\left(\tau_{1}\right) \xi_{1}\left(\tau_{2}\right)\right\rangle d \tau_{1} d \tau_{2} \int_{t}^{1} \int_{\substack{t \\
R_{34}}}^{l} n_{2}\left(\tau_{3}\right) n_{2}\left(\tau_{4}\right)\left\langle\xi_{1}\left(\tau_{3}\right) \xi_{1}\left(\tau_{4}\right)\right\rangle d \tau_{3} d \tau_{4}
\end{aligned}
$$




$$
\begin{gathered}
+\frac{1}{A_{1} B_{1} \varepsilon} \int_{0}^{t} \int_{t}^{1} n_{1}\left(\tau_{1}\right) n_{2}\left(\tau_{3}\right)\left\langle\xi_{1}\left(\tau_{1}\right) \xi_{1}\left(\tau_{3}\right)\right\rangle d \tau_{1} d \tau_{3} \int_{0}^{t} \int_{t}^{1} n_{1}\left(\tau_{2}\right) n_{2}\left(\tau_{4}\right)\left\langle\xi_{1}\left(\tau_{2}\right) \xi_{1}\left(\tau_{4}\right)\right\rangle d \tau_{2} d \tau_{4} \\
+\frac{1}{R_{1} B_{1} \varepsilon} \int_{0}^{t} \int_{t}^{t} n_{1}\left(\tau_{1}\right) n_{2}\left(\tau_{4}\right)\left\langle\xi_{1}\left(\tau_{1}\right) \xi_{1}\left(\tau_{4}\right)\right\rangle d \tau_{1} d \tau_{4} \int_{0}^{t} \int_{t}^{1} n_{1}\left(\tau_{2}\right) n_{2}\left(\tau_{3}\right)\left\langle\xi_{1}\left(\tau_{2}\right) \xi_{1}\left(\tau_{3}\right)\right\rangle d \tau_{2} d \tau_{3} \\
R_{23}
\end{gathered}
$$

where $R_{1234}, R_{12}, \ldots, R_{23}$ are maximally $\varepsilon$-neighboring of $\tau_{1}, \tau_{2}, \tau_{3}, \tau_{4} ; \tau_{1}, \tau_{2} ; \ldots ; \tau_{2}, \tau_{3}$ respectively, and (2.7) has been used to obtain the last result.

By means of $(3.10)$, we can have

$$
\begin{gathered}
(3.13)=\frac{1}{A_{1} B_{1} \varepsilon^{2}} \int_{0}^{t} \int_{0}^{t} n_{1}\left(\tau_{1}\right) n_{1}\left(\tau_{2}\right)\left\langle\xi_{1}\left(\tau_{1}\right) \xi_{1}\left(\tau_{2}\right)\right\rangle d \tau_{1} d \tau_{2}, \\
\int_{t}^{1} \int_{R_{34}}^{1} n_{2}\left(\tau_{3}\right) n_{2}\left(\tau_{4}\right)\left\langle\xi_{1}\left(\tau_{3}\right) \xi_{1}\left(\tau_{4}\right)\right\rangle d \tau_{3} d \tau_{4}+0(\varepsilon) .
\end{gathered}
$$

After using the same procedure for other terms in (3.12), we find

$$
\left\langle\frac{n_{1}^{2}}{A_{1} \varepsilon} \cdot \frac{n_{2}^{2}}{B_{1} \varepsilon}\right\rangle=\left\langle\frac{n_{1}^{2}}{A_{1} \varepsilon}\right\rangle\left\langle\frac{n^{2} 2}{B_{1} \varepsilon}\right\rangle+0(\varepsilon) .
$$

4. THE DENSITY FUNCTION AND THE STRUCTURE OF THE SOLUTION.

In this section we will consider the density function of the solution $\underset{\sim}{x}(t)$ and if we notice that the situation will be simple when $t=0$ or $t=1$, so our main interest is in the case when $t \in(0,1)$.

In order to find the density function of $x(t)$, we first consider $\gamma_{l}(t, w)$ or $n_{1}(t, w)$ and $n_{2}(t, w)$ which are defined by (3.4).

We introduce a new function $\mathrm{p}_{1}\left(\mathrm{v}_{1}, \mathrm{v}_{2}\right)$ such that

$$
\frac{p_{1}}{\sqrt{A_{1} \varepsilon}}, \frac{n_{2}}{\sqrt{B_{1} \varepsilon}}\left(v_{1}, v_{2}\right)=p_{\frac{n_{1}}{\sqrt{A_{1} \varepsilon}}}\left(v_{1}\right) \cdot p_{\frac{n_{2}}{\sqrt{B_{1} \varepsilon}}}\left(v_{2}\right)+p_{1}\left(v_{1}, v_{2}\right)
$$

where $p_{n_{l}}\left(v_{1}\right)$ is the density function of $\frac{n_{1}}{\sqrt{A_{1} \varepsilon}}$ and so on. Because of (3.11) we $\overline{\sqrt{A_{1} \varepsilon}}$

have for $K_{1}, K_{2}=0,1, \ldots$

$$
\begin{aligned}
& \int_{-\infty}^{\infty} \int_{-\infty}^{\infty} v_{1} \mathrm{~K}_{1} v_{2} \mathrm{~K}_{2} \mathrm{p}_{1}\left(\mathrm{v}_{1}, \mathrm{v}_{2}\right) \mathrm{d} \mathrm{v}_{1} \mathrm{~d} \mathrm{v}_{2} \\
& =\int_{-\infty}^{\infty} \int_{-\infty}^{\infty} v_{1} \mathrm{~K}_{1} v_{2} \mathrm{~K}_{2} \frac{\mathrm{p}_{n_{1}}}{\sqrt{\mathrm{A}_{1} \varepsilon}}, \frac{n_{2}}{\sqrt{\mathrm{B}_{1} \varepsilon}}\left(\mathrm{v}_{1}, \mathrm{v}_{2}\right) \mathrm{d} \mathrm{v}_{1} \mathrm{~d} \mathrm{v}_{2}- \\
& \int_{-\infty}^{\infty} \int_{-\infty}^{\infty} v_{1}{ }^{K_{1}} v_{2}{ }^{K_{2}} \underline{p_{n_{1}}}\left(v_{1}\right) \cdot \underline{p_{n_{2}}}\left(v_{2}\right) d v_{1} d v_{2}
\end{aligned}
$$




$$
\begin{aligned}
& =\left\langle\left(\frac{n_{1}}{\sqrt{A_{1} \varepsilon}}\right)^{K_{1}}\left(\frac{n_{2}}{\sqrt{B_{1} \varepsilon}}\right)^{K_{2}}\right\rangle-\left\langle\left(\frac{n_{1}}{\sqrt{A_{1} \varepsilon}}\right)^{K_{1}}\right\rangle\left\langle\left(\frac{n_{2}}{\sqrt{B_{1} \varepsilon}}\right)^{K_{2}}\right\rangle \\
& =0(\varepsilon) .
\end{aligned}
$$

Then from (4.1) we know when $\varepsilon$ is a small number but not zero

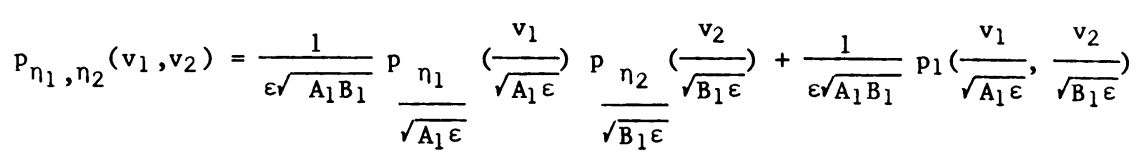

and from (3.3) we have

$$
\begin{aligned}
p_{\gamma_{1}}(v) & =\int_{-\infty}^{\infty} p_{n_{1} n_{2}}(z, v-z) d z \\
& =\frac{1}{\varepsilon \sqrt{A_{1} B_{1}}} \int_{-\infty}^{\infty} \frac{p_{n_{1}}}{\sqrt{A_{1} \varepsilon}}\left(\frac{z}{\sqrt{A_{1} \varepsilon}}\right) \frac{p_{n_{2}}}{\sqrt{B_{1} \varepsilon}}\left(\frac{v-z}{\sqrt{B_{1} \varepsilon}}\right) d z+ \\
& \frac{1}{\varepsilon \sqrt{A_{1} B_{1}}} \int_{-\infty}^{\infty} p_{1}\left(\frac{z}{\sqrt{A_{1} \varepsilon}}, \frac{v-z}{\sqrt{B_{1} \varepsilon}}\right) d z
\end{aligned}
$$

Under the assumtion that the density function of $\underset{\sim}{\alpha(\omega)}$ is $p_{\alpha \beta}(\alpha, \beta)$, we can easily find the density function of $\underset{\sim}{\Psi}(t) \underset{\sim}{\alpha(\omega)}$, that is

$$
\mathrm{p}_{\alpha_{1} \alpha_{2}}\left(\alpha_{1}, \alpha_{2}\right)=\mathrm{p}_{\alpha \beta}(\alpha, \beta)\left|\operatorname{Det} \underset{\sim}{\Psi}(t)^{-1}\right| \mid \underset{\beta}{\left(\begin{array}{l}
\alpha \\
\beta
\end{array}\right)=\underset{\sim}{\Psi}-1(t)\left(\begin{array}{l}
\alpha_{1} \\
\alpha_{2}
\end{array}\right)}
$$

where $\underset{\sim}{\Psi}(t) \underset{\sim}{\alpha}(\omega)=\left(\alpha_{1}(\omega), \alpha_{2}(\omega)\right)^{\mathrm{T}}, \mathrm{P}_{\alpha_{1} \alpha_{2}}$ is the density function of $\alpha_{1}(\omega), \alpha_{2}(\omega)$, and Det $\underset{\sim}{\Psi}(t)^{-1}$ is the determinant of the inverse matrix $\underset{\sim}{\Psi}(t)^{-1}$.

Then the density function of $\alpha_{1}(\omega)$ is

$$
\begin{aligned}
& \mathrm{p}_{\alpha_{1}}\left(\alpha_{1}\right)=\int_{-\infty}^{\infty} \mathrm{p}_{\alpha_{1} \alpha_{2}\left(\alpha_{1}, \alpha_{2}\right) \mathrm{d} \alpha_{2}} \\
& =\int_{-\infty}^{\infty} p_{\alpha \beta}(\alpha, \beta)\left|\operatorname{Det} \underset{\sim}{\Psi}(t)^{-1}\right| \quad\left(\begin{array}{l}
\alpha \\
\beta
\end{array}\right)={\underset{\sim}{\Psi}}^{-1}(t)\left(\begin{array}{l}
\alpha_{1} \\
\alpha_{2}
\end{array}\right) d \alpha_{2} .
\end{aligned}
$$

Because of (3.3) and (3.4) if we assume that $\underset{\sim}{\alpha(\omega)}$ is independent of $\underset{\sim}{\xi}(t, \omega)$ for every $t \quad[0,1]$, then $\alpha_{1}(\omega)$ and $\gamma_{1}(t, \omega)$ will be independent and we have

$$
\begin{aligned}
p_{\alpha_{1}+\gamma_{1}}(w) & =\int_{-\infty}^{\infty} p_{\alpha_{1}}(w-v) p_{\gamma_{1}}(v) d v \\
& =\frac{1}{\varepsilon \sqrt{A_{1} B_{1}}} \int_{-\infty}^{\infty} \int_{-\infty}^{\infty} p_{\alpha_{1}}(w-v) p_{\frac{\eta_{l}}{\sqrt{A_{1} \varepsilon}}} \frac{\left(\frac{z}{\sqrt{A_{1} \varepsilon}}\right) p_{\frac{\eta_{2}}{\sqrt{B_{1} \varepsilon}}}\left(\frac{v-z}{\sqrt{B_{1} \varepsilon}}\right) d z d v}{\varepsilon \sqrt{A_{1} B_{1}}} \int_{-\infty}^{\infty} \int_{-\infty}^{\infty} p_{\alpha_{1}}(w-v) p_{1}\left(\frac{z}{\sqrt{A_{1} \varepsilon}}, \frac{v-z}{\sqrt{B_{1} \varepsilon}}\right) d z d v
\end{aligned}
$$


written as

$$
\begin{aligned}
\frac{1}{\varepsilon \sqrt{A_{1} B_{1}}} \int_{-\infty}^{\infty} \int_{-\infty}^{\infty} p_{\alpha_{1}}(w-v) p_{1}\left(\frac{z}{\sqrt{A_{1} \varepsilon}}, \frac{v-z}{\sqrt{B_{1} \varepsilon}}\right) d z d v \\
=\frac{1}{\varepsilon \sqrt{A_{1} B_{1}}} \int_{-\infty}^{\infty} \int_{-\infty}^{\infty} p_{\alpha_{1}}\left(w-T \sqrt{B_{1} \varepsilon}-S \sqrt{A_{1} \varepsilon}\right) p_{1}(S, T) \varepsilon \sqrt{A_{1} B_{1}} d S d T \\
=\int_{-\infty}^{\infty} \int_{-\infty}^{\infty} p_{\alpha_{1}}\left(w-T \sqrt{B_{1} \varepsilon}-S \sqrt{A_{1} \varepsilon}\right) p_{1}(S, T) d S d T
\end{aligned}
$$

where $S=z / \sqrt{A_{1} \varepsilon}, T=(v-z) / \sqrt{B_{1} \varepsilon}$.

If we can expand $P_{\alpha_{1}}$ into a Taylor series in $S, T$

$$
p_{\alpha_{1}}\left(w-T \sqrt{B_{1} \varepsilon}-s \sqrt{A_{1} \varepsilon}\right)=\sum_{i, j=0}^{\infty} C^{*}{ }_{i j} s^{i} T^{j}
$$

and allow the following analytic procedure, then after using (4.2) we have

$$
(4.7)=\sum_{i, j=0}^{\infty} C^{\star}{ }_{i j} \int_{-\infty}^{\infty} \int_{-\infty}^{\infty} S^{i} T^{j} p_{l}(S, T) d S d T=0(\varepsilon) .
$$

Thus (4.6) becomes

$$
\mathrm{p}_{\alpha_{1}+\gamma_{1}}(w)=\frac{1}{\varepsilon \sqrt{\mathrm{A}_{1} \mathrm{~B}_{1}}} \int_{-\infty}^{\infty} \int_{-\infty}^{\infty} \mathrm{p}_{\alpha_{1}}(w-v) \mathrm{p} \frac{\eta_{1}}{\sqrt{\mathrm{A}_{1} \varepsilon}}\left(\frac{z}{\sqrt{\mathrm{A}_{1} \varepsilon}}\right) \mathrm{p} \frac{\eta_{2}}{\sqrt{\mathrm{B}_{1} \varepsilon}}\left(\frac{\mathrm{v}-z}{\sqrt{\mathrm{B}_{1} \varepsilon}}\right) \mathrm{dzd}+0(\varepsilon)
$$

and from (3.1a) we have

$$
\left.\mathrm{p}_{\mathrm{x}}(\mathrm{x})=\frac{1}{\varepsilon \sqrt{\mathrm{A}_{1} \mathrm{~B}_{1}}} \int_{-\infty}^{\infty} \int_{-\infty}^{\infty} \mathrm{p}_{\alpha_{1}}\left(\mathrm{x}-\delta_{1}-\mathrm{v}\right) \mathrm{p} \frac{\eta_{1}}{\sqrt{\mathrm{A}_{1} \varepsilon}}\left(\frac{\mathrm{z}}{\sqrt{\mathrm{A}_{1} \varepsilon}}\right) \mathrm{p} \frac{n_{2}}{\frac{\sqrt{\mathrm{B}_{1} \varepsilon}}{\sqrt{\mathrm{B}_{1} \varepsilon}}}\right) \mathrm{dzd}+0(\varepsilon)
$$

where $x, \delta_{1}$ are the first component of $\underset{\sim}{x}(t), \underset{\sim}{\delta}(t)$ respectively, and $\mathrm{P}_{x}(x)$ is the density function of $x$.

In order to obtain the asymptotic approximation for $p_{x}(x)$, we need the following facts [see [5]],

$$
\begin{aligned}
& \mathrm{p} \frac{n_{1}}{\sqrt{\mathrm{A}_{1} \varepsilon}}\left(\mathrm{v}_{1}\right)=\frac{1}{\sqrt{2 \pi}} \exp \left(-\frac{\mathrm{v}_{1}}{2}\right)\left[1+\sqrt{\varepsilon} \mathrm{H}_{1}^{\ell_{1}}\left(\mathrm{v}_{1}\right)+\ldots\right] \\
& \mathrm{p} \frac{n_{2}}{\frac{2}{\sqrt{\mathrm{B}_{1} \varepsilon}}}\left(\mathrm{v}_{2}\right)=\frac{1}{\sqrt{2 \pi}} \exp \left(-\frac{\mathrm{v}_{2}}{2}\right)\left[1+\sqrt{\varepsilon} \mathrm{H}_{2}\left(\mathrm{v}_{2}\right)+\ldots\right]
\end{aligned}
$$

where $\mathrm{H}_{1}^{\ell_{1}}, \mathrm{H}_{2}{ }_{2}$ are certain polynomials of degree $\ell_{1}, \ell_{2}$ respectively and the dots denote some polynomials with the order $\varepsilon$.

To substitute (4.9) into $(4.8)$, we obtain

$$
\mathrm{p}_{\mathrm{x}}(\mathrm{x})=\mathrm{I}_{1}+\sqrt{\varepsilon} \mathrm{I}_{2}+\sqrt{\varepsilon} \mathrm{I}_{3}+O(\varepsilon) \text {. }
$$


where

$$
\begin{aligned}
& I_{1}=\frac{1}{2 \pi \varepsilon \sqrt{A_{1} B_{1}}} \int_{-\infty}^{\infty} p_{\alpha_{1}}\left(x-\delta_{1}-v\right) \exp \left[-\frac{1}{2 \varepsilon}\left(\frac{z^{2}}{A_{1}}+\frac{(v-z)^{2}}{B_{1}}\right) d z d v,\right. \\
& \left.I_{2}=\frac{1}{2 \pi \varepsilon \sqrt{A_{1} B_{2}}} \int_{-\infty}^{\infty} \int_{-\infty}^{\infty} p_{\alpha_{1}}\left(x-\delta_{1}-v\right) \exp \left[-\frac{1}{2 \varepsilon}\left(\frac{z^{2}}{A_{1}}+\frac{(v-z)^{2}}{B_{1}}\right)\right] H_{1} \frac{\ell_{1}}{\sqrt{A_{1} \varepsilon}}\right) d z d v, \\
& I_{3}=\frac{1}{2 \pi \varepsilon \sqrt{A_{1} B_{1}}} \int_{-\infty}^{\infty} \int_{-\infty}^{\infty} p_{\alpha_{1}}\left(x-\delta_{1}-v\right) \exp \left[-\frac{1}{2 \varepsilon}\left(\frac{x^{2}}{A_{1}}+\frac{(v-z)^{2}}{B_{1}}\right)\right] H_{2} \ell_{2}\left(\frac{v-z}{\sqrt{B_{1} \varepsilon}}\right) d z d v .
\end{aligned}
$$

From the fact

$$
\frac{1}{2 \pi \varepsilon \sqrt{A_{1} B_{2}}} \exp \left[-\frac{1}{2 \varepsilon}\left(\frac{z^{2}}{A_{1}}+\frac{(v-z)^{2}}{B_{1}}\right)\right] \stackrel{\text { W.C. }}{\longrightarrow} \delta(z, v-z), \quad(\varepsilon \rightarrow 0)
$$

where W.C. denotes weak convergence, and $\delta$ is the Delta function; we have

$$
\begin{aligned}
\lim _{\varepsilon \rightarrow 0} I_{1} & =\int_{-\infty}^{\infty} \int_{-\infty}^{\infty} p_{\alpha_{1}}\left(x-\delta_{1}-v\right) \delta(z, v-z) d z d v \\
& =p_{\alpha_{1}}\left(x-\delta_{1}\right)
\end{aligned}
$$

and if we can show that $I_{2}$ and $I_{3}$ are bounded as $\varepsilon+0$, then in (4.10) we can keep the first term as the main term.

In fact for $I_{2}$ if we set $\ell_{1}=0$ or $H_{1}=a_{0}=$ constant, we have

$$
\begin{aligned}
& \lim I_{2}=\lim a_{0} I_{1}=a_{0} p_{\alpha_{1}}\left(x-\delta_{1}\right), \\
& \varepsilon \rightarrow 0 \quad \varepsilon \rightarrow 0
\end{aligned}
$$

and for $\ell_{1}=1,2, \ldots$ or for $I_{3}$ where $\ell_{2}=0,1, \ldots$ we can use the similar way to obtain the conclusion.

Thus as $\varepsilon+0$ we can consider $\eta_{1}(t, \omega)$ and $\eta_{2}(t, \omega)$ to be independent, and we obtain the asymptotic formula for $x(t)$

$$
\begin{aligned}
\mathrm{p}_{\mathrm{x}}(\mathrm{x}) & =\frac{1}{2 \pi \varepsilon \sqrt{\mathrm{A}_{1} \mathrm{~B}_{1}}} \int_{-\infty}^{\infty} \int_{-\infty}^{\infty} \mathrm{p}_{\alpha_{1}}\left(\mathrm{x}-\delta_{1}-v\right) \exp \left[-\frac{1}{2 \varepsilon}\left(\frac{z^{2}}{\mathrm{~A}_{1}}+\frac{(v-z)^{2}}{B_{1}}\right)\right] \mathrm{dzd} v \\
& =\frac{1}{\sqrt{2 \pi \varepsilon\left(\mathrm{A}_{1}+\mathrm{B}_{1}\right)}} \int_{-\infty}^{\infty} \mathrm{p}_{\alpha_{1}}\left(\mathrm{x}-\delta_{1}-\mathrm{v}\right) \exp \left[-\frac{v^{2}}{2 \varepsilon\left(\mathrm{A}_{1}+\mathrm{B}_{1}\right)}\right] \mathrm{dv},
\end{aligned}
$$

where $\delta_{1}$ and $\mathrm{p}_{\alpha_{1}}$ can be evaluated in terms of (3.1b) and (4.5) respectively.

The asymototic formula for $y(t)$, which is the second component of the solution $\mathrm{x}(\mathrm{t})$, can be obtained in a similar way.

By means of these asymptotic formulas we can find the structure of the solution. If we recall the functional form (3.1a) of the solution $\underset{\sim}{x}(t)$, and notice that $\underset{\sim}{\Psi}(t) \underset{\sim}{\alpha(w)}$ is the solution of the equation

$$
\frac{d \underset{\sim}{d}(t)}{d t}=\underset{\sim}{A}(t) \underset{\sim}{x}(t), \quad D_{1} \underset{\sim}{x}(1)+D_{2} \underset{\sim}{x}(1)=\underset{\sim}{u}(\omega) ;
$$


$\underset{\sim}{\delta(t)}$ is the solution of the deterministic equation

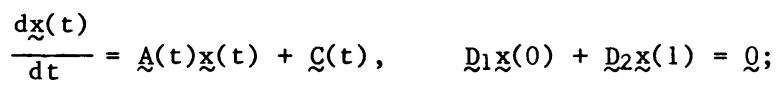

and that $\mathfrak{X}(t, \omega)=\left(\gamma_{1}(t, \omega), \gamma_{2}(t, \omega)\right)^{T}=\left(n_{1}(t, w), n_{3}(t, \omega)\right)^{T}+\left(n_{2}(t, w), n_{4}(t, w)\right)^{T}$ is the solution of the equation

$$
\frac{\mathrm{d}_{\sim}(t)}{d t}=\underset{\sim}{A}(t) \underset{\sim}{x}(t)+\underset{\sim}{B}(t) \xi(t, w), \quad{\underset{\sim}{1}}_{1} \underset{\sim}{x}(0)+\underset{\sim}{D_{2}} \underset{\sim}{x}(1)=\underset{\sim}{0},
$$

where $\underset{\sim}{0}$ is a $2 \times 1$ vector with zero components, then we can say that the solution $\mathrm{x}(t)$ has such kind of structure that it consists of three functions, the first one is

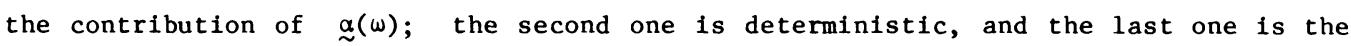
contribution of $\underset{\sim}{\xi}(t, \omega)$. When $\varepsilon \rightarrow 0$ every component of the last function has the normal distribution with the mean value zero and the variance $\varepsilon\left(A_{1}+B_{1}\right)$, and it can be divided into two independent normal random processes.

5. THE JOINT DENSITY FUNCTION AND THE EXAMPLE.

If the matrices $\mathrm{D}_{1}, \underset{\sim}{\mathrm{D}_{2}}$ in the boundary condition (2.1b) have the forms

$$
\mathrm{D}_{1}=\left(\begin{array}{cc}
\mathrm{d}_{11} & \mathrm{~d}_{12} \\
0 & 0
\end{array}\right), \quad \mathrm{D}_{2}=\left(\begin{array}{ll}
0 & 0 \\
\mathrm{~d}_{21} & \mathrm{~d}_{22}
\end{array}\right)
$$

then we can find the joint density function of the solution $\underset{\sim}{x}(t)=(x(t), y(t))^{T}$. In fact under the assumption (5.1), the solution is

$$
\underset{\sim}{x}(t)=\underset{\sim}{\Psi}(t)\left[\underset{\sim}{\alpha}(\omega)+\underset{\sim}{\delta}(t)+\mathfrak{L}^{*}(t, \omega)\right]
$$

where

$$
\begin{aligned}
& \delta^{*}(t)=\left(\begin{array}{c}
\delta_{1}^{*}(t) \\
\delta_{2}^{*}(t)
\end{array}\right)=\int_{0}^{t} \mathrm{~m}_{1}(\tau) \mathrm{d} \tau \\
& \mathscr{L}^{*}(t, \omega),=\left(\begin{array}{c}
\gamma_{1}^{*}(t, \omega) \\
\gamma_{2}^{*}(t, w)
\end{array}\right)=\left(\begin{array}{l}
t \\
\int_{0}^{t}\left(n_{1}^{*}(\tau) \xi_{1}(\tau, \omega)+r_{1}^{*}(\tau) \xi_{2}(\tau, \omega)\right) d \tau \\
\int_{t}^{1}\left(n_{2}^{*}(\tau) \xi_{1}(\tau, \omega)+r_{2}^{*}(\tau) \xi_{2}(\tau, \omega)\right) d \tau
\end{array}\right),
\end{aligned}
$$

and

$$
\begin{aligned}
& {\underset{\sim}{\Psi}}^{-1}(\tau) \underset{\sim}{\mathcal{C}}(\tau)=\left(\begin{array}{c}
\mathrm{m}_{1}(\tau) \\
-\mathrm{m}_{2}(\tau)
\end{array}\right)
\end{aligned}
$$

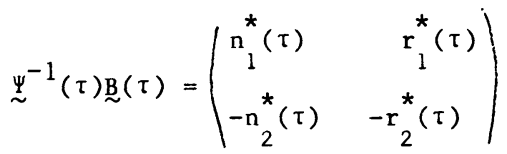


If we know the density function of $\mathfrak{L}^{\star}(\tau, \omega)$, then

$$
p_{\alpha+\chi^{*}}\left(w_{1}, w_{2}\right)=\int_{-\infty}^{\infty} p_{\alpha \beta}\left(w_{1}-\alpha, w_{2}-\beta\right) p_{\chi^{*}}(\alpha, \beta) d \alpha d \beta,
$$

where $p_{\alpha \beta}, P_{\Upsilon^{*}}$ are the density functions of $\underset{\sim}{\alpha(\omega)}$ and $\Upsilon^{*}(t, \omega)$ respectively, and the independence assumption of $\underset{\sim}{\alpha(\omega)}$ and $\underset{\sim}{\xi}(t, \omega)$ has been used again.

But compare (5.4) with (3.4), we can find the fact that $\eta_{1}, \eta_{2} ; \gamma_{1}^{*}, \gamma_{2}^{*}$ have the similar forms except that the functions $n_{1}, r_{1}, n_{2}, r_{2}$ have been repalced by $\mathrm{n}_{1}^{*}, \mathrm{r}_{1}^{*}, \mathrm{n}_{2}^{*}, \mathrm{r}_{2}^{*}$ respectively.

It follows that

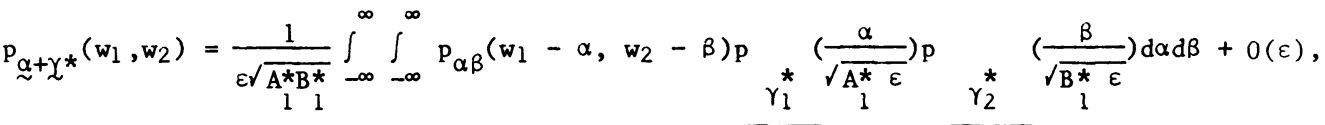

$$
\begin{aligned}
& \frac{r_{1}^{*}}{\sqrt{A^{*} \varepsilon}}{ }_{1}^{A_{1}^{*} \varepsilon} \frac{r_{2}^{*}}{\sqrt{B^{*} \varepsilon}}
\end{aligned}
$$

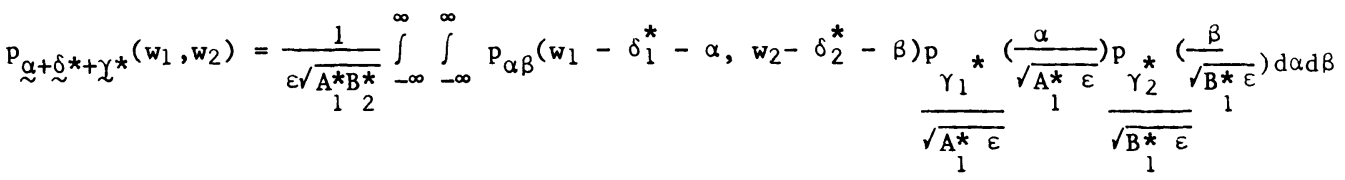

$$
\begin{aligned}
& +O(\varepsilon) \text {. }
\end{aligned}
$$

In a similar way we have the asymptotic formula

$$
\begin{aligned}
& \mathrm{p}_{\underset{\sim}{\alpha+\delta} \underset{\sim}{*}+\gamma_{\sim}^{*}}\left(\mathrm{w}_{1}, \mathrm{w}_{2}\right)=\frac{1}{2 \pi \varepsilon \sqrt{\mathrm{A}_{1}^{*} \mathrm{~B}_{1}^{*}}} \int_{-\infty}^{\infty} \int_{-\infty}^{\infty} \mathrm{p}_{\alpha \beta}\left(\mathrm{w}_{1}-\delta_{1}^{*}-\alpha, \mathrm{w}_{2}-\delta_{2}^{*}-\beta\right) \\
& \exp \left[-\frac{1}{2 \varepsilon}\left(\frac{\alpha^{2}}{\mathrm{~A}^{*}}+\frac{\beta^{2}}{\mathrm{~B}^{*}}\right)\right] \mathrm{d} \alpha \mathrm{d} \beta
\end{aligned}
$$

and

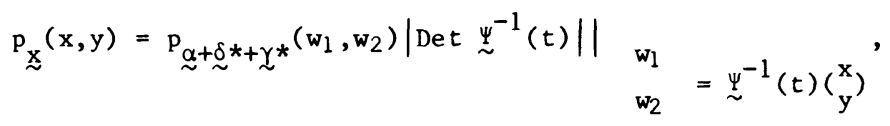

where $\mathrm{p}_{x}$ is the density function of $\underset{\sim}{x}(t)=(x(t), y(t))^{\mathrm{T}}$; and $\delta_{\sim}^{*}(t)=\left(\delta_{1}^{*}(t), \delta_{2}^{*}(t)\right)^{\mathrm{T}}, \mathrm{A}_{1}^{*}, \mathrm{~B}_{1}^{*}$ can be evaluated by $(5.3),(5.5),(5.6)$ and the formula which likes (3.5) but the functions $n_{1}, n_{2}, r_{1}, r_{2}$ need to be replaced by $\mathrm{n}_{1}^{*}, \mathrm{n}_{2}^{*}, \mathrm{r}_{1}^{*}, \mathrm{r}_{2}^{*}$ respectively.

Now we consider the first example

$$
\begin{aligned}
& \left(\begin{array}{l}
\dot{x} \\
y
\end{array}\right)=\left(\begin{array}{rr}
0 & 1 \\
-2 & -3
\end{array}\right)\left(\begin{array}{l}
x \\
y
\end{array}\right)+\left(\begin{array}{rll}
1 & 0 \\
-1 & 1
\end{array}\right) \begin{array}{l}
\xi_{1}(t, w) \\
\xi_{2}(t, \omega)
\end{array}+\left(\begin{array}{l}
1 \\
0
\end{array}\right), \\
& x(0)=\alpha(\omega), \quad y(1)=\beta(\omega),
\end{aligned}
$$


where $\xi_{1}(t, \omega), \xi_{2}(t, \omega)$ are independent weakly correlated processes with correlation length $\varepsilon,\left\langle\xi_{1}(t, \omega)\right\rangle=0,\left\langle\xi_{2}(t, \omega)\right\rangle=0$,

$$
R_{\xi_{1}}(\tau, \tau)=1, \quad R_{\xi_{2}}(\tau, \tau)=1, \quad \text { and } \quad p_{\alpha \beta}(\alpha, \beta)=\frac{1}{2 \pi} \exp \left(-\frac{\alpha^{2}+\beta^{2}}{2}\right) \text {. }
$$

It is easy to find that

$$
\begin{gathered}
\underset{\sim}{\Phi}(t)=\left(\begin{array}{ll}
\phi_{11}(t) & \phi_{12}(t) \\
\phi_{21}(t) & \phi_{22}(t)
\end{array}\right)=\left(\begin{array}{cc}
2 e^{-t}-e^{-2 t} & e^{-t}-e^{-2 t} \\
-2 e^{-t}+2 e^{-2 t} & -e^{-t}+2 e^{-2 t}
\end{array}\right), \\
\underset{\sim}{\Psi^{-1}}(t)=e^{2 t}\left(\begin{array}{cc}
-1+2 e^{-t}-1+e^{-t} \\
A
\end{array}\right) \\
A=\left(2 \phi_{22}(1)-\phi_{21}(1)\right)+2 e^{-t}\left(\phi_{21}(1)-\phi_{22}(1)\right) \\
B=\left(2 \phi_{22}(1)-\phi_{21}(1)\right)+e^{-t}\left(\phi_{21}(1)-\phi_{22}(1)\right)
\end{gathered}
$$

and

$$
\begin{aligned}
&\left(\begin{array}{rr}
n_{1}^{*} & r_{1}^{*} \\
-n_{2}^{*} & -r_{2}^{*}
\end{array}\right)=e^{t}\left(\begin{array}{cc}
1 & 1-e^{t} \\
\phi_{21}(1)-\phi_{22}(1) & e^{t}\left(2 \phi_{22}(1)-\phi_{21}(1)\right)+\left(\phi_{21}(1)-\phi_{22}(1)\right)
\end{array}\right) \\
&\left(\begin{array}{r}
m_{1} \\
-m_{2}
\end{array}\right)=e^{t}\left(\begin{array}{cc}
2 & e^{t} \\
e^{t}\left(2 \phi_{22}(1)-\phi_{21}(1)\right)+2\left(\phi_{21}(1)-\phi_{22}(1)\right)
\end{array}\right)
\end{aligned}
$$

Then

$$
\begin{aligned}
A_{1}^{*}(t)=2\left[\frac{e^{4 t}}{4}-\frac{2 e^{3 t}}{3}+e^{2 t}-\frac{7}{12}\right] & \\
B_{1}^{*}(t) & =2\left[\frac{e^{4}\left(2 \phi_{22}(1)-\phi_{21}(1)\right)^{2}}{4}+\frac{2 e^{3}\left(2 \phi_{22}(1)-\phi_{21}(1)\right)\left(\phi_{21}(1)-\phi_{22}(1)\right)}{3}\right. \\
+ & e^{2\left(\phi_{21}(1)-\phi_{22}(1)\right)^{2}-e^{2 t}\left(\phi_{21}(1)-\phi_{22}(1)\right)^{2}} \\
\left.-\frac{2 e^{3 t}\left(2 \phi_{22}(1)-\phi_{21}(1)\right)\left(\phi_{21}(1)-\phi_{22}(1)\right)}{3}\right] &
\end{aligned}
$$

and

$$
\mathrm{p}_{\mathrm{xy}}(\mathrm{x}, \mathrm{y})=\frac{0.0309425 \mathrm{e}^{3 t}}{2 \sqrt{\left(1+\varepsilon A_{1}^{*}(t)\right)\left(1+\varepsilon B_{1}^{*}(t)\right)}} \exp \left\{-\left[\frac { 1 } { 2 ( 1 + \varepsilon A _ { 1 } ^ { \star } ( t ) ) } \left(1.5+e^{t}(2 x+y-2)\right.\right.\right.
$$




$$
\begin{aligned}
& \left.-e^{2 t}(x+y-0.5)\right)^{2}+\frac{1}{2\left(1+\varepsilon B_{1}^{*}(t)\right)}\left(-1-0.3678794 e^{t}(2 x+y-2)\right. \\
& \left.\left.\left.+0.2706705 e^{2 t}(x+y-0.5)\right)^{2}\right\}\right\},
\end{aligned}
$$

where

$$
\begin{aligned}
& A_{1}^{*}(t)=2\left[\frac{e^{4 t}}{4}-\frac{2 e^{3 t}}{3}+e^{2 t}-\frac{7}{12}\right] \\
& B_{1}^{*}(t)=2\left[\frac{2}{3}-e^{2(t-1)}+\frac{4}{3} e^{3(t-1)}-e^{4(t-1)}\right]
\end{aligned}
$$

The results of evaluating (5.13) when $t=0.5, y=-0.6860$ appear in Figure 1 for $\varepsilon=0$ (solid curve), $\varepsilon=0.2$ (longer dashed curve), and $\varepsilon=0.4$ (shorter dashed curve).

In Figures 2, 3, and 4 we show some level curves of the density function p:

$$
\begin{aligned}
& \mathrm{p}=0.04, \quad \mathrm{t}=0.5, \quad \varepsilon=0,0.2,0.4 \quad \text { (Figure 2); } \\
& \mathrm{p}=0.005, \quad \mathrm{t}=0.5, \quad \varepsilon=0,0.2,0.4 \quad \text { (Figure } 3 \text { ); } \\
& t=0.5, \quad \varepsilon=0.2, \quad p=0.01,0.03,0.05 \quad \text { (Figure 4) }
\end{aligned}
$$

The second example is

$$
\left\{\begin{array}{l}
\ddot{x}+3 \dot{x}+2 x=f(t, \omega) \\
x(0)=\alpha(\omega), \quad \dot{x}(1)=B(\omega)
\end{array}\right.
$$

where $f(t, \omega)$ is a weakly correlated process with the correlation length $\varepsilon,\langle f(t, w)\rangle=0$ and $R_{f}(t, t)=1$.

We introduce a new function $y(t)=\dot{x}(t)$ and rewrite (5.14)

$$
\begin{aligned}
& \left(\begin{array}{l}
\dot{x} \\
y
\end{array}\right)=\left(\begin{array}{rr}
0 & 1 \\
-2 & -3
\end{array}\right)\left(\begin{array}{l}
x \\
y
\end{array}\right)+\left(\begin{array}{l}
0 \\
1
\end{array}\right) f(t, w) \\
& \left(\begin{array}{ll}
1 & 0 \\
0 & 0
\end{array}\right)\left(\begin{array}{l}
x(0) \\
y(0)
\end{array}\right)+\left(\begin{array}{ll}
0 & 0 \\
0 & 1
\end{array}\right)\left(\begin{array}{l}
x(1) \\
y(1)
\end{array}\right)=\left(\begin{array}{l}
\alpha(\omega) \\
\beta(\omega)
\end{array}\right) .
\end{aligned}
$$

This equation has the same fundamental matrices as in the first example, then we have

$$
\underset{\sim}{\sim}(t)=\left(\begin{array}{cc}
\phi_{11}(t)-\phi_{12}(t) \frac{\phi_{21}(1)}{\phi_{22}(1)} & \frac{\phi_{12}(t)}{\phi_{22}(1)} \\
\phi_{21}(t)-\phi_{22}(t) \frac{\phi_{21}(1)}{\phi_{22}(1)} & \frac{\phi_{22}(t)}{\phi_{22}(1)}
\end{array}\right)
$$

where $\phi_{i j}$ are the components of $\underset{\sim}{\Phi}(t)$ and can be evaluated by $(5.12)$. From (3.1) we can obtain 


$$
\underset{\sim}{\underline{X}(t)}=\underset{\sim}{\Psi}(t)\left(\begin{array}{l}
\alpha(\omega) \\
\beta(\omega)
\end{array}\right)+\left(\begin{array}{l}
\gamma_{1}(t, \omega) \\
\gamma_{2}(t, \omega)
\end{array}\right)
$$

where

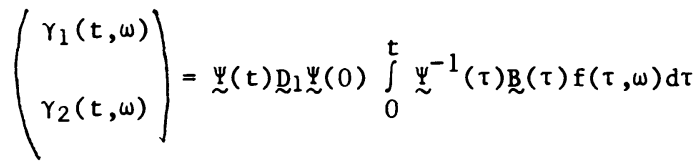

$$
\begin{aligned}
& -\underset{\sim}{\Psi}(t) D_{2} \underset{\sim}{\Psi}(1) \int_{t}^{1} \underset{\sim}{\Psi} \Psi^{-1}(\tau) \underset{\sim}{B}(\tau) f(\tau, \omega) \mathrm{d} \tau \\
& =\left(\begin{array}{ll}
\phi_{11}(t)-\phi_{12}(t) \frac{\phi_{21}(1)}{\phi_{22}(1)} \\
\phi_{21}(t)-\phi_{22}(t) & \frac{\phi_{21}(1)}{\phi_{22}(1)}
\end{array}\right) \int_{0}^{t}\left(e^{\tau}-e^{2 \tau}\right) f(\tau, \omega) d \tau \\
& -\left(\begin{array}{c}
\phi_{12}(t) \\
\phi_{22}(t)
\end{array}\right) \frac{1}{\phi_{22}(1)} \int_{t}^{1}\left[\left(2 \phi_{22}(1)-\phi_{21}(1)\right) e^{2 \tau}+\left(\phi_{21}(1)-\phi_{22}(1)\right) e^{\tau}\right] f(\tau, \omega) d \tau
\end{aligned}
$$

Then the solution $x(t)$ of the equation (5.14) has the structure

$$
\begin{aligned}
& x(t)=\frac{1}{\phi_{22}(1)}\left\{\left[\phi_{11}(t) \phi_{22}(1)-\phi_{12}(t) \phi_{21}(1)\right] \alpha(\omega)-\phi_{12}(t) \beta(\omega)\right\}+ \\
& \frac{1}{\phi_{22}(1)}\left\{\left[\phi_{11}(t) \phi_{22}(1)-\phi_{12}(t) \phi_{21}(1)\right] \int_{0}^{t}\left(e^{\tau}-e^{2 \tau}\right) f(\tau, \omega) d \tau-\right. \\
& \left.\phi_{12}(t) \int_{t}^{1}\left[\left(2 \phi_{22}(1)-\phi_{21}(1)\right) e^{2 \tau}+\left(\phi_{21}(1)-\phi_{22}(1)\right) e^{\tau}\right] f(\tau, \omega) d \tau\right\} .
\end{aligned}
$$

The first term is the contribution of $(\alpha(\omega), \beta(\omega))^{\mathrm{T}}$; and the second term, which contains two integrals, is the contribution of $f(t, \omega)$.

When the correlation length $\varepsilon \rightarrow 0$, the second term approaches normal with the mean value zero and the variance $\varepsilon\left(A_{1}+B_{1}\right)$, where

$$
\begin{aligned}
& A_{1}=2\left[\phi_{11}(t)-\phi_{12}(t) \frac{\phi_{21}(1)}{\phi_{22}(1)}\right]^{2} \int_{0}^{t}\left(e^{\tau}-e^{2 \tau}\right)^{2} R_{f}(\tau, \tau) d \tau \\
&=2\left[\phi_{11}(t)-\phi_{12}(t) \frac{\phi_{21}(1)}{\phi_{22}(1)}\right]^{2}\left[\frac{e^{4 t}-1}{4}+\frac{e^{2 t}-1}{2}-\frac{2\left(e^{3 t}-1\right)}{3}\right] \\
& B_{1}=2 \frac{\phi_{12}^{2}(t)}{\phi_{22}^{2}(1)} \int_{t}^{1}\left[\left(2 \phi_{22}(1)-\phi_{21}(1)\right) e^{2 \tau}+\left(\phi_{21}(1)-\phi_{22}(1)\right) e^{\tau}\right]^{2} R_{f}(\tau, \tau) d \tau \\
&=2 \frac{\phi_{12}^{2}(1)}{\phi_{22}^{2}(1)}\left[\frac{\left(2 \phi_{22}(1)-\phi_{21}(1)\right)^{2}}{4} e^{(4-4 t)}=\frac{\left(\phi_{21}(1)-\phi_{22}(1)\right)^{2}}{2} e^{(2-2 t)}+\right. \\
&\left.\frac{2}{3}\left(2 \phi_{22}(1)-\phi_{21}(1)\right)\left(\phi_{21}(1)-\phi_{22}(1)\right) e^{(3-3 t)}\right] .
\end{aligned}
$$




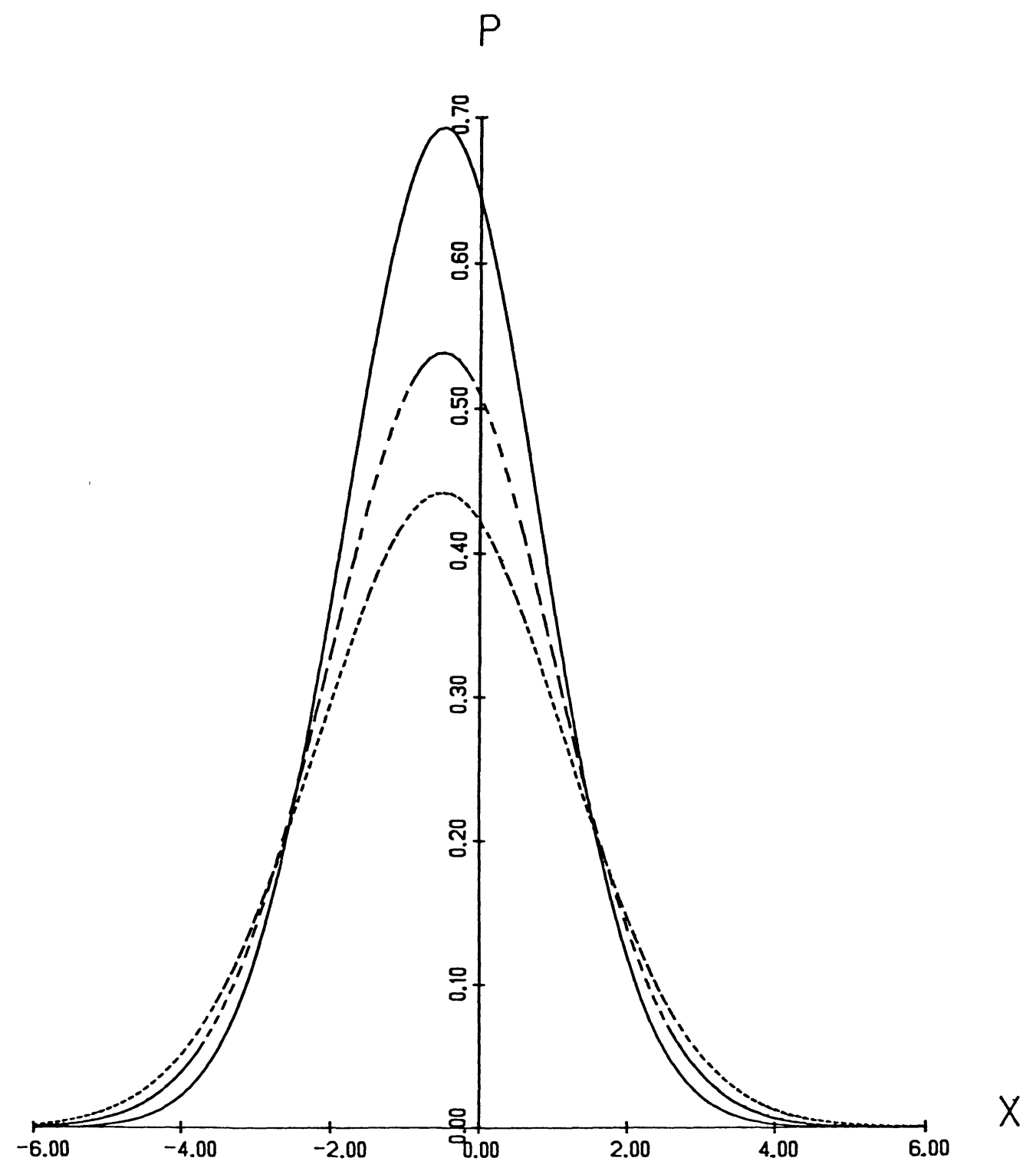

$P=10.0 \times$ DENSITY FUNCTION $P(X,-0.6860)$

FIGURE 1 


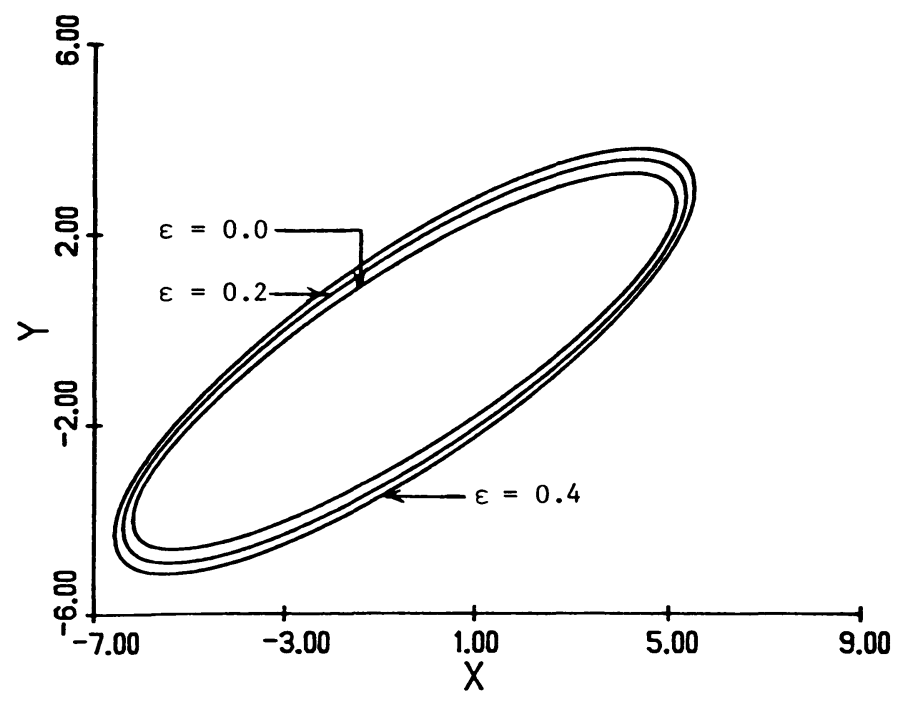

FIGURE 3

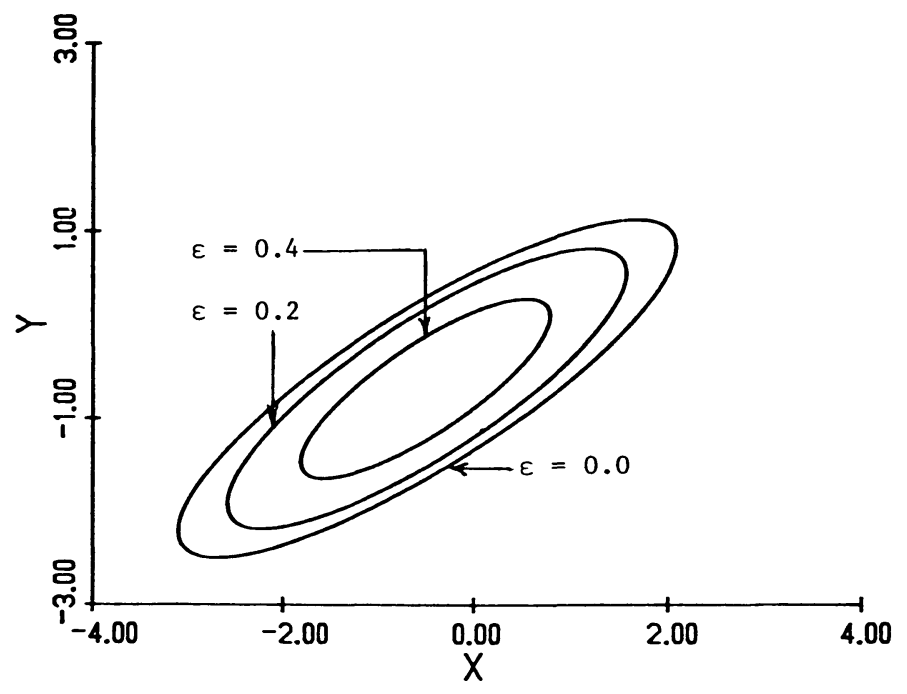

FIGURE 2 


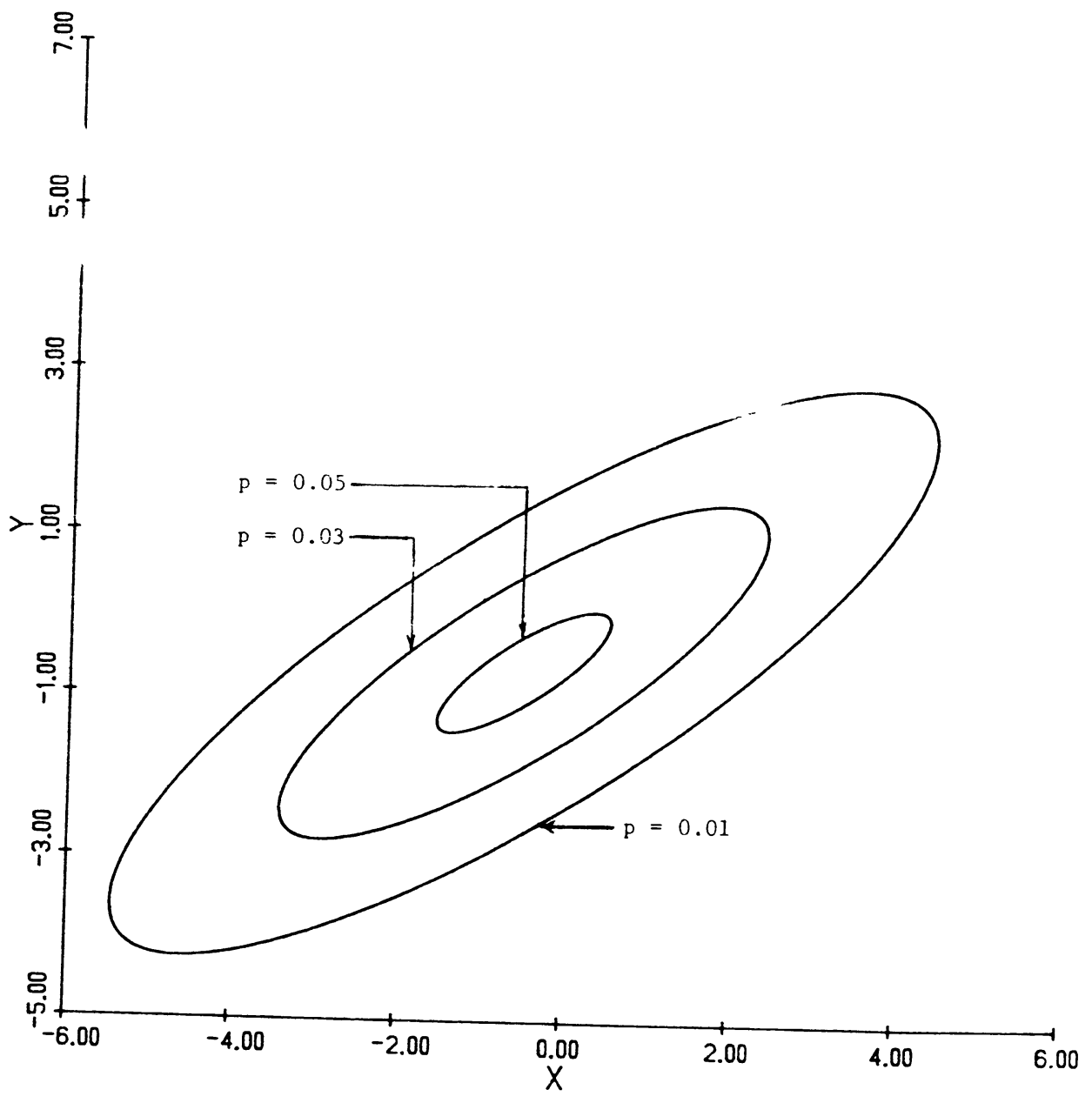

FIGURE 4

REFERENCES

1. UHLENBECK, G.E. and ORNSTEIN, L.S. On the Theory of Brownian Motion, Phys. Rev., $\underline{36}$ (1930), 823-841.

2. BOYCE, W.E. Stochastic Nonhomogeneous Sturm-Liouville Problems, J. Franklin Inst., 282 (1966), 206-215.

3. PURKERT, W. and vom SCHEIDT, J. Randwertprobleme mit Schwach Korrelierten Prozessen als Koeffizienten, Transactions of Eighth Prague Conference on Information Theory, Statistical Decision Functions, and Random Processes (1978), Volume B, 107-118.

4. vom SCHEIDT, J. and PURKERT, W. Limit Theorems for Solutions of Stochastic Differential Equation Problems, Int. J. Math. and Math. Sci., 3 (1980), 113-149.

5. BOYCE, W. E. and XIA, N.M. The Approach to Normality of the Solutions of Random Boundary and Eigenvalue problems with Weakly Correlated Coefficients, 0. Appl. Math., 40 (1983), 419-445.

6. PURKERT, W. and vom SCHEIDT, J. Ein Grenzverteilungssatz fur Stochastische Eigenwertprobleme, ZAMM 59 (1979), 611-623. 


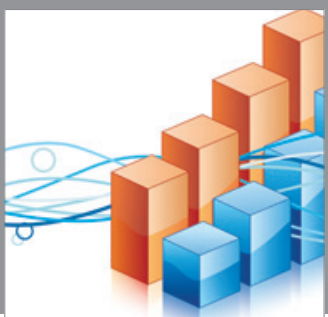

Advances in

Operations Research

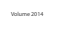

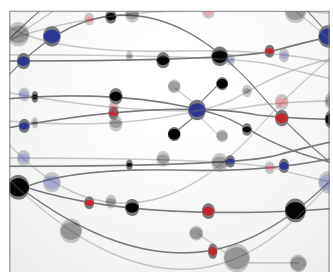

\section{The Scientific} World Journal
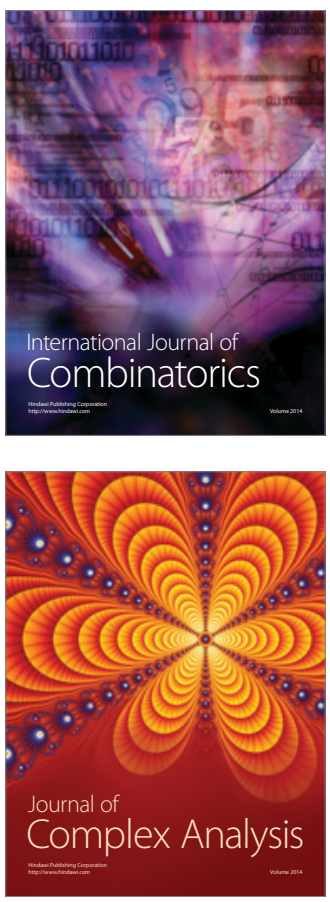

International Journal of

Mathematics and

Mathematical

Sciences
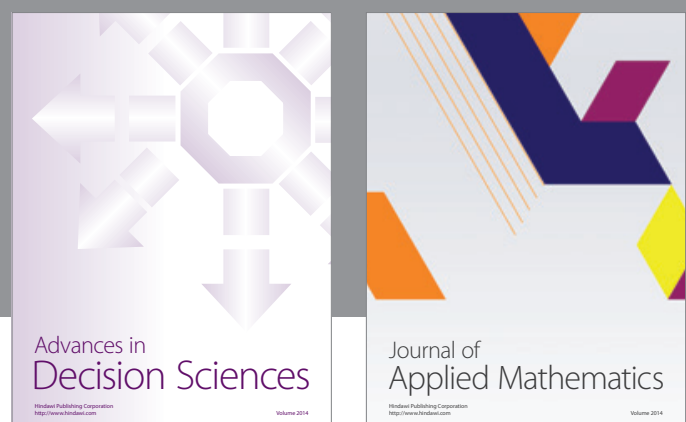

Journal of

Applied Mathematics
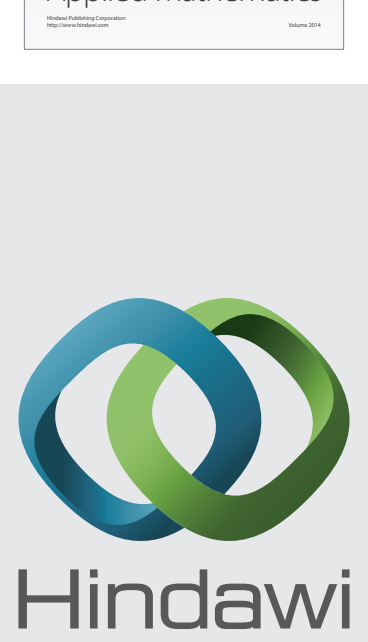

Submit your manuscripts at http://www.hindawi.com
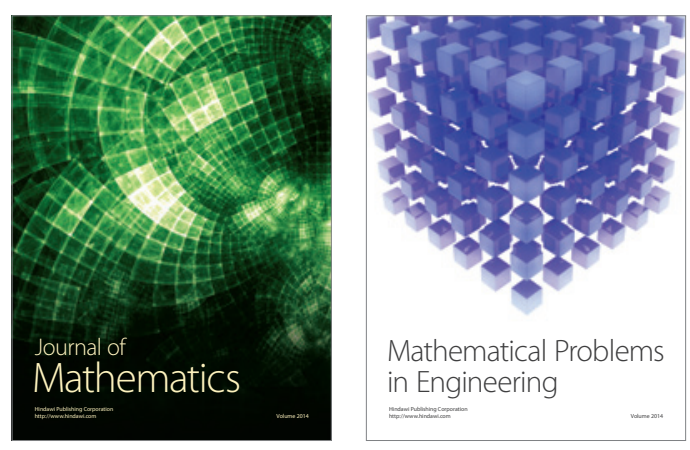

Mathematical Problems in Engineering
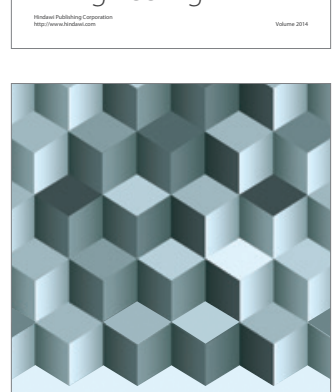

Journal of

Function Spaces
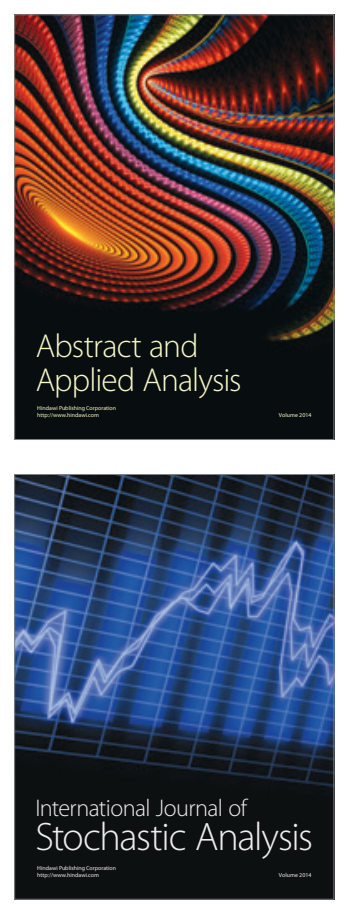

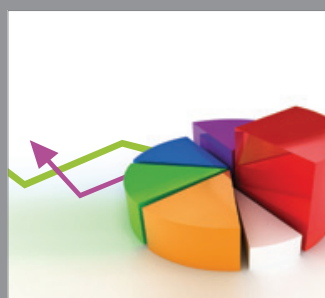

ournal of

Probability and Statistics

Promensencen
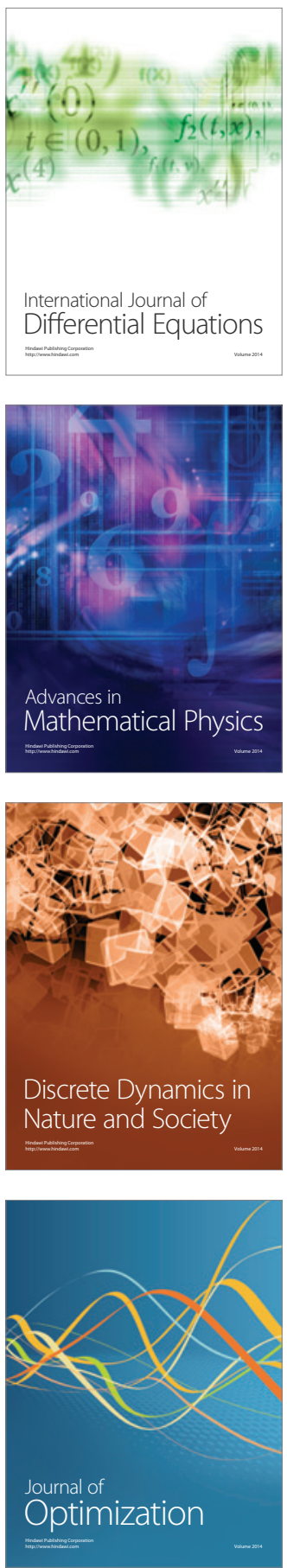